Portland State University

PDXScholar

Winter 4-28-2014

\title{
Effects of the First Language on Japanese ESL Learners' Answers to Negative Questions
}

Kosuke Kanda

Portland State University

Follow this and additional works at: https://pdxscholar.library.pdx.edu/open_access_etds

Part of the First and Second Language Acquisition Commons Let us know how access to this document benefits you.

\section{Recommended Citation}

Kanda, Kosuke, "Effects of the First Language on Japanese ESL Learners' Answers to Negative Questions" (2014). Dissertations and Theses. Paper 1704.

https://doi.org/10.15760/etd.1703

This Thesis is brought to you for free and open access. It has been accepted for inclusion in Dissertations and Theses by an authorized administrator of PDXScholar. Please contact us if we can make this document more accessible: pdxscholar@pdx.edu. 


\author{
Effects of the First Language \\ on Japanese ESL Learners’ Answers \\ to Negative Questions
}

by

Kosuke Kanda

A thesis submitted in partial fulfillment of the requirements for the degree of

\author{
Master of Arts \\ in \\ Teaching English to Speakers of Other Languages
}

Thesis Committee:

Susan Conrad, Chair

Kimberly Brown

Suwako Watanabe

Portland State University

2014 


\begin{abstract}
This study investigates how Japanese learners of English respond to English negative questions. Previous research has reported that Japanese learners of English make errors in yes/no responses to English negative questions due to the first language (L1) influence (Kang \& Lim-chang, 1998; Takashima, 1989). From the perspective of L1 influence, there are two learning pitfalls: different functions of the yes/no response and different interpretations of negative questions. Both of these influences were examined in this study.
\end{abstract}

This study involved 8 Japanese learners of English, 4 females and 4 males, attending Portland State University (PSU). In order to elicit data that reflect the effect of Japanese English Language Teaching (ELT), the subjects were chosen so that at the time of data elicitation, they had less than 6 months of experience in an English-speaking environment. In addition, all the participants had English instruction in Japan at least through high school.

In order to see how the L1 influenced their yes/no answers to negative questions, I used two data elicitation methods: an oral interview with a native speaker and a retrospective protocol analysis of the interview. The results indicated the following: First, the participants appeared to respond English negative questions fairly consistently with the English norm. Deviation was observed only when a negative question had a negative expected answer. Particularly, the stronger the expectation for a negative answer was, the more likely it was that the negative question elicited an incorrect yes/no response. Secondly, the participants interpreted the polarity of the expected answer based on the Japanese norm. With the help of context, they usually interpreted the stimulus sentence 
correctly. However, when an expected answer was ambiguous for any reason, the participants interpreted the stimulus sentence as having a negative expected answer, which is the default interpretation for Japanese negative questions.

This study shows that the influence of the L1 on answers to negative questions requires complex analysis. That is, superficially the participants appeared to answer questions correctly, but a deeper analysis revealed that they still relied on an L1 interpretation norm. 


\section{Dedication}

I dedicate this thesis to my father and mother who have supported me throughout

the process. I will always appreciate all they have done for me. I also dedicate this work

and give special thanks to my "little brother" Qoo, who had always given me morale

support and ended his short life wishing for my success. May you find peace and

happiness up there. 
Table of contents

Abstract............................................................................

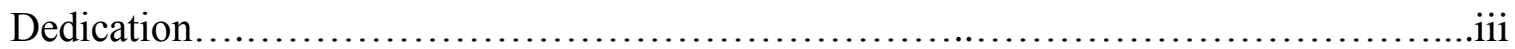

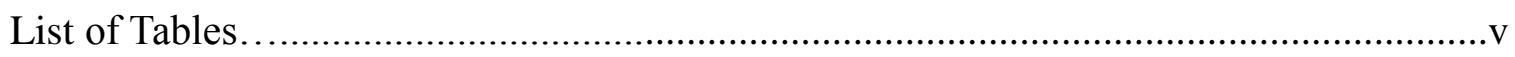

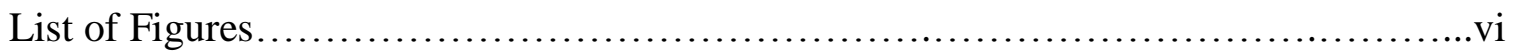

\section{Chapter 1}

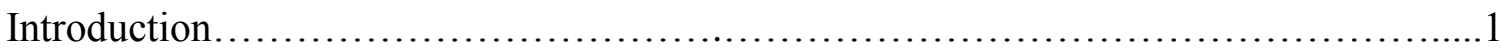

Chapter 2

Literature Review...............................................................4

\section{Chapter 3}

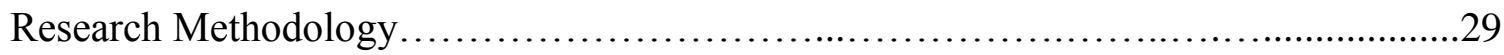

\section{Chapter 4}

Results and Discussion....................................................41

\section{Chapter 5}

Conclusion....................................................................

References................................................................ 71

Appendix
Interview Scenario.......................................................... 74 


\section{List of Tables}

Table 1

Default question-and-answer sequence in the two languages ........................17

Table 2

The demographic information of the participants

Table 3

Stimulus Sentences.

Table 4

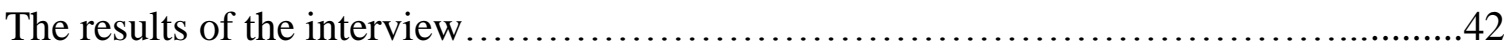

Table 5

The general results of the retrospective protocol analysis...........................45

Table 6

The individual results of the retrospective analysis per participant 


\section{List of Figures}

Figure 1

Function of the Japanese response morphemes...................................

Figure 2

Degree of the questioner's assumptions in Japanese.............................. 15

Figure 3

Possible performance patterns of Japanese learners of English......................20 


\section{Chapter 1: Introduction}

Since the publication of Lado's influential book Linguistics across Cultures in 1957, the role of the first language (L1) in second language acquisition has been examined from a variety of angles. The theoretical assumption of the original Contrastive Analysis Hypothesis was that the greater the linguistic difference between the L1 and the target language (TL), the greater the likelihood of interference. This assumption has been modified, however, and the field's current understanding of L1 influence is that transfer can now come about through both similarity and difference (Odlin, 1989).

Although myriad studies have been conducted about L1 influence, there still is a surprising level of confusion in the field concerning when, where, in what form, and to what extent (Ellis, 1985; Jarvis, 2000). One area of confusion is the degree to which L1 influence accounts for learners' interlanguage (IL) production errors. Ellis (1985) summarized studies on L1 influence and reported that levels of L1-induced errors range from an almost negligible $3 \%$ to a striking $51 \%$. Apart from which linguistic feature one looks into, this discrepancy is attributable to the differences in experimental design among different studies as well as a lack of well-defined and broadly-accepted criteria for establishing which grammatical utterance is the result of language transfer (Ellis, 1994).

Transfer-based language learning theory has also received much criticism. Evidence against transfer theory often comes from the comparison of ILs among learners with different backgrounds (Odlin, 1989). For example, Dulay and Burt (1974) researched the acquisition of morphemes by Spanish and Chinese L1 learners 
and found that there seems to be a natural sequence irrespective of different L1s. Their study was criticized for its research design and data interpretation, and more importantly, they do not think of TL input and instruction as a crucial variable in L2 learning.

TL input as well as output, however, is often considered to play a key role in language learning. For EFL learners, the picture of the TL acquisition process is slightly different from naturalistic and ESL situations because the TL input is usually limited to a classroom, so EFL learners have more reliance on their L1 and/or learned patterns (Takashima, 1989).

The present study is designed to shed light on Japanese learners of English. Today, Japanese EFL education is attempting to shift from the traditional grammar-focused curriculum to a more communicative one so that students are better able to participate in a globalized society. In order to provide more sophisticated English language teaching, it is necessary to understand how Japanese learners of English who have taken English classes through high school behave in a practical setting.

This study focuses on responses to negative interrogatives, one of the grammar points that Japanese learners of English have trouble with. I have heard many Japanese learners of English respond to English NQs in the same way that they do in Japanese, for example:

(1) A: Don't you have an umbrella? B: No, I do.

In the reply to A's question, the Japanese learner (B) uttered a sentence which does not 
comply with the English prescriptive norm, i.e., either "Yes, I do" or "No I don't." The question arises here as to what the knowledge source behind the Japanese learner's answer is; specifically, is this a transfer error?

There are a growing number of studies devoted to examining the errors English learners make when answering NQs, and it is generally found that difficulties arise for learners from several L1 groups: Japanese (M. M. Akiyama, 1979; M. Akiyama, 1976; Kang \& Lim-Chang, 1998; Takashima, 1989); Korean (Baik \& Shim, 1993; Kang \& Lim-Chang, 1989); Chinese (Yat-Shing, 1974); and Polynesian (Lane, 1993). What these languages share in common is that their $\mathrm{Y} / \mathrm{N}$ response is radically different from that of English. Therefore, the studies are designed to consider the issue in relation to the learners' $L 1$.

In the present study, I seek to investigate the knowledge source Japanese learners of English resort to when replying to English NQs. Because L1 seems to be the most likely explanation for the frequently observed errors among Japanese learners, this study uses contrastive analysis. Describing how the two languages are different, I attempt to increase our understanding of why Japanese learners make errors in responding to English NQs. The study makes a new contribution by creating a situation that elicits more contextualized and spontaneous answers from learners than previous studies have and examines responses to negative questions in complete, contextualized question-and-answer sequences. It considers the effect of the L1 and language instruction from analysis of the learners' answers, but also uses a retrospective analysis to consider learners' understanding of the questions and context and to investigate their thinking processes. 


\section{Chapter 2: Literature Review}

This chapter presents a review of the literature and considers some of the key issues necessary for analyzing why Japanese learners of English often fail to successfully acquire English negative questions (NQs). First, I present the literature describing the two languages' different answering systems and the implications I have drawn from these studies. Second, some of the empirical studies that are devoted to pinpointing the knowledge source in responding to English NQs are presented. At the end of this chapter, the research questions for this research study are discussed.

\section{Differences between the two languages}

\section{Functions of a Y/N response}

As of today, there are several research studies that attempt to describe Japanese and English linguistic systems with respect to answering NQs (Akiyama, 1979; Baik \& Shim, 1993; Pope, 1976; Kang \& Lim-Chang, 1998). Although there remains a certain level of confusion regarding a specific rule of response for both English and Japanese, these studies describe important characteristics that may account for second language learners' difficulty.

In an early study, Pope (1976) separated world languages into two types based on their answering systems: positivity/negativity and agreement/disagreement. English belongs to the former category, where yes/no $(\mathrm{Y} / \mathrm{N})$ answers correspond with the positivity/negativity of the proposition that follows the answer. In other words, the two response morphemes, yes and no, are the manifestation of the positivity/negativity of the addressee's statement. 
Japanese, on the other hand, uses the agreement/disagreement system in which $\mathrm{Y} / \mathrm{N}$ answers signal agreement/disagreement with the proposition in the question. According to conventional grammar, the system uses two morphemes, hai and iie in responding to a question. Martin (1962) states, "the words hai and iie are used to mean 'what you've said is correct' and 'what you've said is incorrect,' respectively" (p. 364). Therefore, hai will be chosen if the questioner's and addressee's propositions match, and iie if they do not.

Simply put, the two different answering systems can be described in terms of what element(s) to consider in choosing yes or no (Akiyama, 1979). In the English system, it is only the polarity of the addressee's statement that is relevant in deciding Y/N. The relevant information in the Japanese system, on the other hand, is the relationship between the polarity of the questioner's proposition and that of the addressee's.

Despite the fact that the two languages employ different answering systems, it is almost always the case that the morphemes hai and yes, and iie and no behave identically. This consistency is attributable to their behavior in response to positive questions. Compare the following examples:

(2) English

A: Do you like baseball?

B: Yes, I do. / No I don't.

Japanese

A: yakyuu ga sukidesu-ka?

baseball like

'Do you like baseball?'

(Note that Japanese is a pro-drop language, where the subject of a sentence is not required in conversation) 

B: Hai, sukidesu. / iie,sukidewa arimasen
yes like no like not
'Yes, I do. / No, I don't.'

As the Japanese example indicates, hai corresponds with yes, and iie with no. When a question is positive, the morpheme hai and iie are always parallel to yes and no because agreeing with a positive stimulus sentence derives a positive statement in response, and vice versa. That is, it is reasonable to regard hai and iie as the counterparts of yes and no. For the sake of simplicity, yes is presented as a translation equivalent of hai, and no that of iie.

This account does not hold true, however, when a speaker puts negation in a question. The following is a replication of the example sentences in (2) with negation added:

(3) English

A: Don't you like baseball?

B: Yes, I do. / No I don't.

Japanese

A: yakyuu ga sukidewa-nai no desuka?

baseball like-not that be

'Is it the case that you don't like baseball?'

B: Hai sukidewa-arimasen. / iie, sukidesu.

Yes, like-not no like

‘*Yes, I don't. / *No, I do.'

Contrary to the responses in (2), the Japanese response hai and iie in (3) do not coincide with the English counterparts. If the addressee likes baseball, iie is selected in Japanese despite the fact the addressee's proposition is positive. Likewise, hai is chosen when the addressee does not like baseball. When a question is structured negatively, hai is followed by a negative statement answer and iie by a positive statement answer. Therefore, hai and iie in the case of (3) can best be translated as no 
and yes in English.

This mismatch of how Japanese $\mathrm{Y} / \mathrm{N}$ responses behave between (2) and (3) takes place because of the proposition in Japanese stimulus sentences. Figure 1 presents a schematic for this point. Because the morpheme hai signals concordance between the questioner's and addressee's propositions, responding hai to the stimulus sentence means 'I don't like baseball.' Similarly, iie indicates discordance between interlocutors' propositions, thereby indicating that the addressee likes baseball. That is, Japanese response morphemes have two translation equivalents based on the polarity of the proposition in question: both hai and iie can be translated as either yes or no.

Figure 1: Function of the Japanese response morphemes

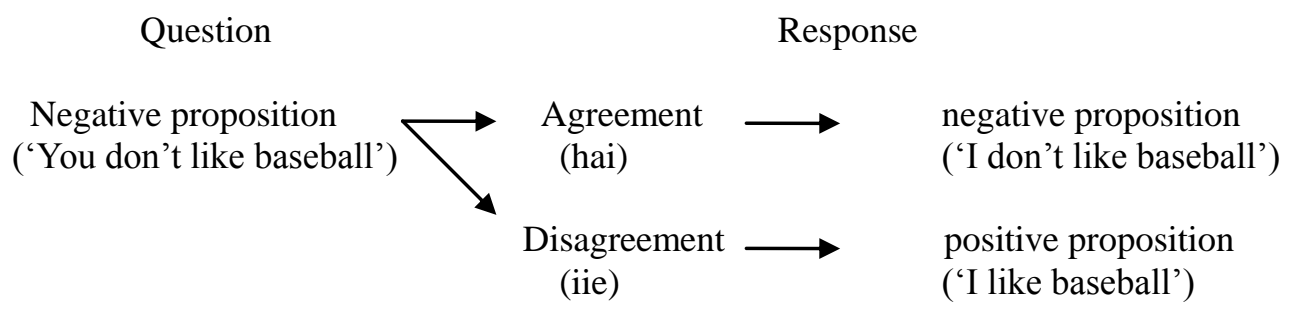

Notice that the Japanese NQ in (3) does not form the translation equivalent of the English counterpart. Despite the fact that its highest clause is structured positively, the stimulus sentence in (3) is considered as a NQ by Japanese native speakers. The following section presents the structure of NQs in each language. 


\section{Structures of negative questions}

Just as the $\mathrm{Y} / \mathrm{N}$ responses are different, the structures of NQs are also different between the two languages. Negative questions have a presuppositional nature where a questioner has a bias towards either positive or negative polarity in both English and Japanese (Bublitz, 1981: Duskova, 1981; Kuno, 1973; Morita, 1988; Nagao. 2005; Teramura, 1984); however, the two languages encode the bias in syntactically different ways.

In English, the bias, namely the expected answer, is not always expressed through the syntactic form, and therefore, there are often times that an expected answer is ambiguous. Consider the following English NQ cited in Dusvoka (1981):

(4) Can't you read?

Dusvoka argues that when the question in (4) is addressed to a child trying to decipher a piece of writing, the questioner expects a negative response, i.e., the question has a negative expected answer. On the other hand, the polarity of the expected answer changes to positive if the identical sentence is addressed to adults who have not wiped their feet despite a sign to do so. That is, the expected answer in English is often dependent on the context in which the NQ is given.

In Japanese a syntactically similar type of NQ is possible, and it can receive different answers depending on the context. For example, the NQ Yome-masen ka? (Can't they read? ) may receive a response, "Hai, yomemasen" (literally, "Yes, they can't.") Alternatively, if the questioner's belief is a positive proposition (e.g., educated adults can read), Yome-masen ka? can be answered with "Hai, yomemasu" (Yes, they can). However, researchers have found that most Japanese NQs differentiate the 
questioner's expected answer by embedding it in a subordinate clause (Kuno, 1973; Morita, 1988; Teramura, 1984). As represented in the example (3), many Japanese NQs are structured in the no/n desu 'it is that ...' pattern (Kuno, 1973; Morita, 1988; Teramura, 1984), and this construction is used when a speaker attempts to confirm the questioner's proposition (Morita, 1988). For example, if questioners hold a negative assumption towards a certain topic, e.g., whether an addressee can swim, they will phrase the question as follows:

(5) Oyogenai no desuka?

Swim-can-not that be

'Is it the case that you cannot swim?'

As the question in (5) indicates, the questioner's assumption is embedded in the subordinate clause (hereafter referred to as the proposition of the question). By phrasing the proposition negatively, the question implies that the questioner is expecting that the addressee cannot swim. On the contrary, if questioners expect that the addressee can swim, they embed a positive proposition into the question:

(6) Oyogeru no desuka?

Swim-can that be

'Is it the case that you can swim?'

The notable difference in NQs between the two languages is the syntactic form. Whereas the syntactic form of English NQs takes the interrogative with a negative in its highest clause, Japanese NQs have a negative morpheme only in a subordinate clause. Although the Japanese NQ in (5) is not a NQ, syntactically speaking, because its highest clause is phrased positively, Japanese speakers consider it a NQ because it contains a semantic negative in the proposition. That is, syntactic negation in the main 
clause is not a necessary component of Japanese NQs.

\section{Variation in the response system in English}

Although in the previous sections, the difference of the $\mathrm{Y} / \mathrm{N}$ responses between the two languages were presented in a clear-cut manner, the account does not hold true for every discourse situation in both languages. A rather extensive study conducted by Nagao (2005) describes the complexity of situations in English. Nagao looked into the authentic response patterns of 22 native English speakers and found variations in their responses to certain NQs. Contrary to the taught rule - i.e., that yes is always followed by a positive proposition and no by a negative proposition variation was found when the polarity of the expected answer was negative and when the pragmatic functions were testing a new assumption or seeking agreement on a negative assumption. Following are some of the NQs that elicited variation cited in Nagao (2005, p. 50-51):

(7) a. Do you not like Katie?

b. You're not having fun, are you?

c. You don't like him.

7(a) and 7(b) are two different types of negative interrogative in structure, but both have a negative expected answer. The difference between the two NQs lies in the pragmatic function; the pragmatic function of 7(a) is testing a new assumption while that of 7(b) is seeking confirmation. $7(\mathrm{c})$ is not a question syntactically or suprasegmentally, but when used to seek confirmation, it functions as an interrogative and elicits a Y/N response. Quite intriguingly, there were more participants whose $\mathrm{Y} / \mathrm{N}$ 
response did not match with the taught rule than those whose $\mathrm{Y} / \mathrm{N}$ response matched for all the NQs in (7). Nagao found that, regardless of the syntactic form of an interrogative, variation is possible when the polarity of the expected answer is negative and when the pragmatic function was testing a new assumption or seeking agreement on a negative assumption.

Furthermore, Nagao's research also indicates that no to disagree with a negative assumption is much more common than yes to agree with a negative assumption. Compare the following two scripts used in Nagao (the capitalized words express emphasis, p. 52):

(8) a. S: You're not having fun, are you?

A: ( ), I AM. I AM having fun.

b. S: You're not going with Amy, are you?

A: ( ), I'm not. I'm going with Nancy.

In his research study, the participants were asked to put either yes or no in the blank. Both of the stimulus sentences in (8) have a negative expected answer as expressed in structure, i.e., the positive tag question elicits a negative answer. The difference lies in the addressee's response; whereas the question in $8(\mathrm{a})$ has a positive tag, the one in 8(b) has a negative tag. Although the practiced rule would produce yes for 8(a) and no for 8(b), the results of the study show that many participants (13 out of 22) did not give a "correct" response in $8(a)$ whereas all the participants gave a correct response in 8(b).

Although Nagao does not discuss this point in depth, this tendency could be attributable to the complexity of yes as a response to NQs. It is generally accepted that there is a tendency in English to use yes in agreement rather than disagreement (Baik 
\& Shim, 1993; Pope, 1976; Kang \& Lim-Chang, 1998). This tendency does not pose confusion when a question is positive. When negative, however, the response yes behaves as disagreement to the speaker's assumption, thereby contradicting its common use. Pope (1976) suggests this category, positive disagreement, is the most difficult and marked answer, noting that when yes is used as disagreement, speakers normally repeat the tag in the answer to achieve successful communication. Using yes with disagreement may be so marked that the participants in Nagao's (2005) study felt that a yes answer followed by a negative proposition was unacceptable.

\section{Variation in the Japanese response system}

The Japanese $\mathrm{Y} / \mathrm{N}$ response to NQs also allows for variation depending on discourse patterns (Angles et al, 2000; Kuno, 1973; Martin, 1962; Shibatani, 1972; Takashima, 1989). Kuno (1973) correctly adds that there are cases in Japanese where Y/N responses behave identically to their English counterparts. Compare the following sentences from Kuno (parts of the English translation for these examples are modified from the original work to keep consistency throughout this study):

(9) A: Kinoo gakkoo ni ikimasen desita ka? yesterday school to go-not did 'Did you not go to school yesterday?'

B: Hai, ikimasen desita yes go-not did '*Yes, I did not go.'

Iie, ikimashita.

no went

'*No, I did' 
(10) A: Kinoo gakko ni itta $\mathrm{n}$ zya arimasen ka? yesterday school to went that be-not

'Isn't it the case that you went to school yesterday?'

B: Hai, ikimashita yo.

yes went

'Yes, I did.'

Iie, ikimasen desita yo.

no went-not

'No, I didn't' $\quad$ (p.274, 275)

The two stimulus sentences are similar in form but elicit different responses; while hai and iie in (9) correspond with English no and yes, respectively, they are used just like English yes and no in (10). Notice that the way the two stimulus sentences are structured is different in spite of the fact that the responding statement in each example refers to the same proposition (whether the addressee went to school or not).

The key to understanding the discrepancy between the responses in (9) and (10) lies in noticing the position of the negative morpheme masen in the stimulus sentences. Comparing the two stimulus sentences in (9) and (10), we see that the negative morpheme is placed inside the proposition that the addressee responds to in (9) while in (10) it is outside the proposition. As presented above, the deciding factor in choosing Japanese $\mathrm{Y} / \mathrm{N}$ is whether the propositions between the interlocutors match or not. The difference in responses between (9) and (10) reveals that the presence of the negative morpheme outside the proposition does not give semantic negativity to the proposition, and therefore, the response morphemes in (10) superficially correspond with the English counterparts (i.e., agreement morpheme hai followed by a positive proposition). 
A question arises here as to the role of the negative morpheme in the main clause, as in the stimulus sentence (10). As observed above, there are two slots that a negative morpheme can fill: the main clause and subordinate clause. Thus, it is possible to have two negative morphemes in a question. Compare the following two NQs:
(11) a. yakyuu ga sukidewanai no desuka? baseball like-not that be 'Is it the case that you don't like baseball?'
b. yakyuu ga sukidewanai no dewa arimasenka?

baseball like-not tha be-not

'Isn't it the case that you don't like baseball?'

The two sentences in (11) have the same proposition (that the addressee does not like baseball) but are different in the structure of the main clause. Kuno (1973) argues that when a question is structured in the no/n desu pattern, the negative morpheme in the main clause plays a pragmatic function of expectation for an agreement. That is, the degree of the questioner's assumption is stronger in 11(b) than in 11(a). as manifested by the negative morpheme in the main clause. Figure 2 summarizes the degree of the questioner's assumption based on syntactic structure.

If a questioner does not have any bias towards either polarity of the proposition, the question in Japanese is structured by only adding a question particle $-k a$ at the end of the sentence. When questioners have an expected answer, they usually phrase the question using the no/n desu construction, putting either a positive or negative proposition in the subordinate clause. The stronger assumption questioners have about the proposition being correct, the more likely that they are to phrase the question with a negative morpheme in its highest clause. 
Figure 2: Degree of the questioner's assumption in Japanese

\begin{tabular}{|c|c|}
\hline \multirow{3}{*}{$\begin{array}{l}\text { Assumption that the } \\
\text { positive proposition } \\
\text { is true }\end{array}$} & $\begin{array}{l}\text { Yakyuu ga sukinano dewa arimasenka? } \\
\text { Isn't it the case that you like baseball? }\end{array}$ \\
\hline & $\begin{array}{l}\text { Yakyuu ga sukina no desuka? } \\
\text { Is it the case that you like baseball? }\end{array}$ \\
\hline & $\begin{array}{l}\text { Yakyuu ga suki desuka } \\
\text { Do you like baseball? }\end{array}$ \\
\hline \multirow{2}{*}{$\begin{array}{l}\text { Assumption that the } \\
\text { negative proposition } \\
\text { is true }\end{array}$} & $\begin{array}{l}\text { Yakyuu ga suki dewanai no desuka } \\
\text { Is it the case that you don't like baseball? }\end{array}$ \\
\hline & $\begin{array}{l}\text { Yakyuu ga sukidewa nai no dewa arimasenka? } \\
\text { Isn't it the case that you don't like baseball? }\end{array}$ \\
\hline
\end{tabular}

A word of caution is necessary here. As has been observed by many researchers (Akiyama, 1976; Akiyama, 1979; Angles et al, 2000; Kuno, 1973; Martin, 1962; Shibatani, 1972; Takashima, 1989), Japanese NQs by default have a negative proposition. When Japanese native speakers talk about NQs, it is always NQs below neutral in Figure 1 because the negative morpheme in NQs above neutral does not convey semantic negativity. Therefore, to most Japanese NQs, the response morpheme hai 'yes' will be used for introducing a negative statement answer, and iie 'no' for a positive statement answer.

\section{Summary of the differences between the two languages}

As has been described so far, there are several differences between the two 
languages with respect to negative questions. The differences include not just how the response morphemes function but also the default structure and interpretation of the negative questions.

First of all, the two languages are different in how the response morphemes function. The English Y/N response is consistent with the polarity of the answerer's proposition while the Japanese $\mathrm{Y} / \mathrm{N}$ signals agreement or disagreement between the addressee's and questioner's propositions. The different answering systems mean that English cannot have a yes followed by a negative tag, or vice versa, while it is possible in Japanese because the Japanese response morpheme does not directly affect the polarity of the tag. The Y/N responses in each language conflict when the stimulus sentence contains a negative proposition.

Differences also exist in the typical form of the negative questions themselves (the stimulus sentences). The difference concerns whether the polarity of the proposition in question is explicitly stated. Because Japanese $\mathrm{Y} / \mathrm{N}$ responses show agreement/disagreement, the polarity of the expected answer is more often clearly stated in the stimulus sentence.

Ambiguity in the questioner's expected answer is not appreciated in Japanese because it blurs the target with which the response morpheme agrees. For example, an English NQ like “can’t you read?” causes trouble for a Japanese Y/N response. Because this stimulus sentence does not specify the speaker's expected answer, it is unclear which response morpheme to choose. Therefore, Japanese NQs have to contain a proposition of either polarity, which is usually achieved through syntactic structure, intonation, and/or context. The same stimulus sentence does not cause 
trouble in English because the $\mathrm{Y} / \mathrm{N}$ morpheme in English can be uniquely chosen based on the polarity of the response statement.

Lastly, the two languages are also different in the default definition of NQs. The Japanese NQs are questions whose expected answers have negative polarity. There are many instances of Japanese questions which have a negative morpheme but are not considered NQs. The negative morpheme in these questions does not affect the proposition but serves a pragmatic function of an expectation for agreement. Having a negative morpheme, then, does not necessarily mean that the question is interpreted as a NQ in Japanese. Therefore, the polarity of the proposition in Japanese NQs by default is negative. English NQs, on the other hand, do not have a default polarity for the expected answer. Although there is a way to express the polarity of the questioner's expected answer syntactically, the polarity of the English NQs is basically dependent on the context.

These differences between the two languages result in a totally different set of question and answer sequences. Table 1 presents a default negative question-and-answer sequence for each language for the proposition 'you like baseball'.

Table 1. Default question-and-answer sequence in the two languages

\begin{tabular}{|c|c|c|c|}
\hline & Structure of the NQ & & onse \\
\hline & (polarity of the proposition) & $\mathrm{Y} / \mathrm{N}$ & Underlying intention \\
\hline ENG & Don't you like baseball? & Yes & I like baseball. \\
\hline & (positive/negative) & No & I don't like baseball. \\
\hline JPN & Is it the case that you don't like baseball? & No & I like baseball \\
\hline & (negative) & Yes & I don't like baseball. \\
\hline
\end{tabular}


A word of caution is necessary because the sequence in Table 1 displays only the default use of NQs. In both languages, there are many factors that influence the interpretation of the NQs such as intonation and context. Nevertheless, it is important to keep the default patterns in each language in mind because, as will be discussed later, the difference is claimed to be the source of the many errors learners of each language tend to make. In the following section, how these differences can potentially influence L2 acquisition will be discussed.

\section{Potential miscommunication caused by L1 influence}

The research studies previously conducted on how Japanese learners of English respond to NQs have mainly considered the issue in relation to the L1 (Akiyama, 1979; Kang \& Lim-Chang, 1998; Nagao, 2005; Takashima, 1989). Looking into the differences between Japanese and English, we can see two potential learning pitfalls: the interpretation of the stimulus sentence and the different answering systems. If we consider the mental process of Japanese learners of English, there are 8 possible performance patterns in a question-and-answer sequence. I have summarized the patterns in Figure 3.

If learners have a native-like proficiency, their mental process most likely takes patterns 1 and 5, where both interpretation of the stimulus sentence and choice of response morpheme are correct. If L1 influences learners' performance, a learner's performance would fall into patterns 4 and 6 . In both of these patterns, the stimulus sentence is interpreted as having a negative expected answer - the case where the response morphemes in each language are not parallel. In error pattern 4 , the L1 
influences the learner in two ways. If this error type is observed, it is reasonable to believe that the degree of L1 influence is quite high. If patterns 2, 3 or 7 are observed, the picture of NQ acquisition can be said to take a quite complicated process because this pattern does not fall into either Japanese or English pattern. 
Figure 3: Possible performance patterns of Japanese learners of English

Pitfall $1 \quad$ Pitfall 2

Interpretation of the SS Different $\mathrm{Y} / \mathrm{N}$ response

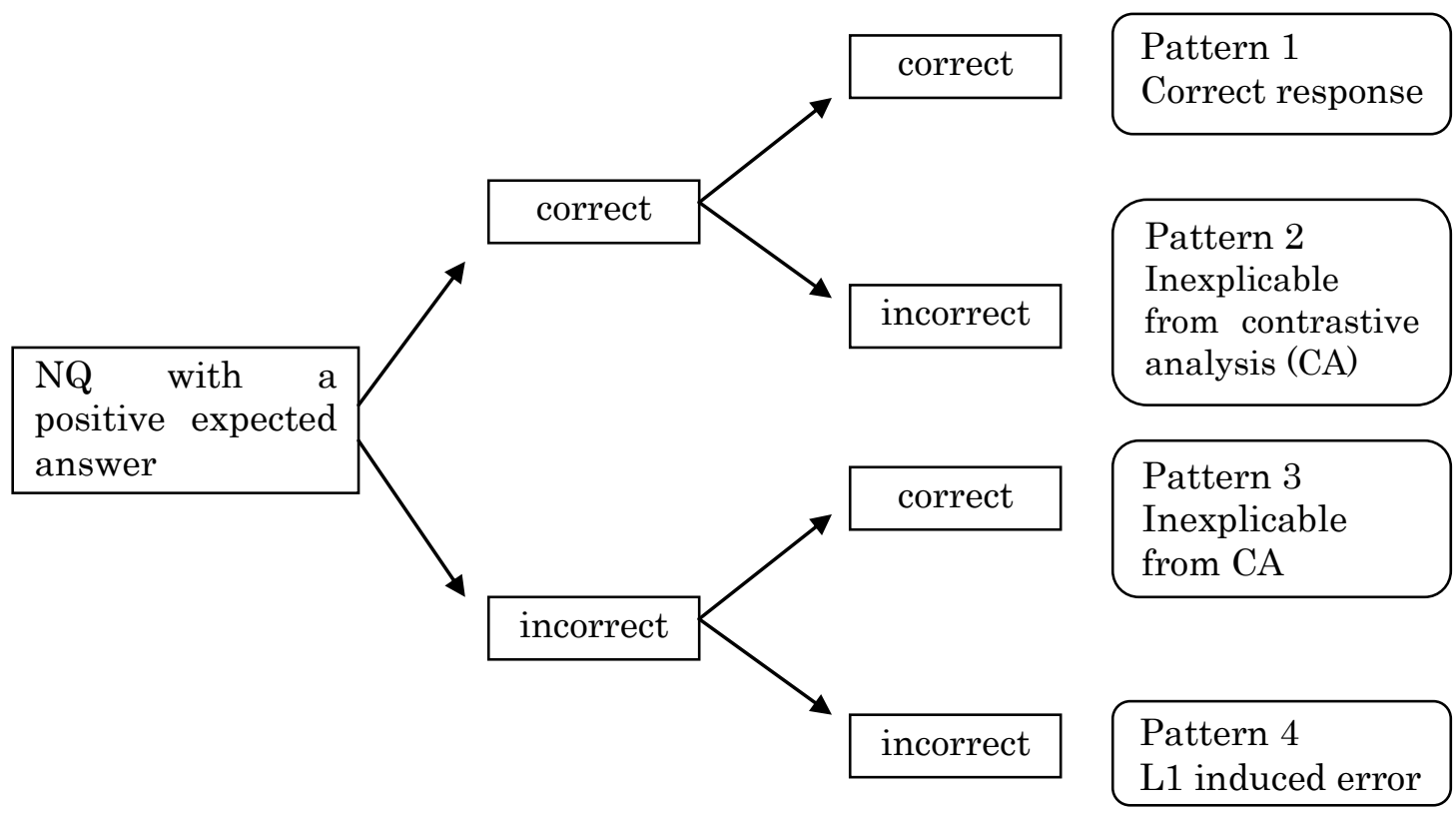

Pitfall $1 \quad$ Pitfall 2

Interpretation of the SS Different $\mathrm{Y} / \mathrm{N}$ response

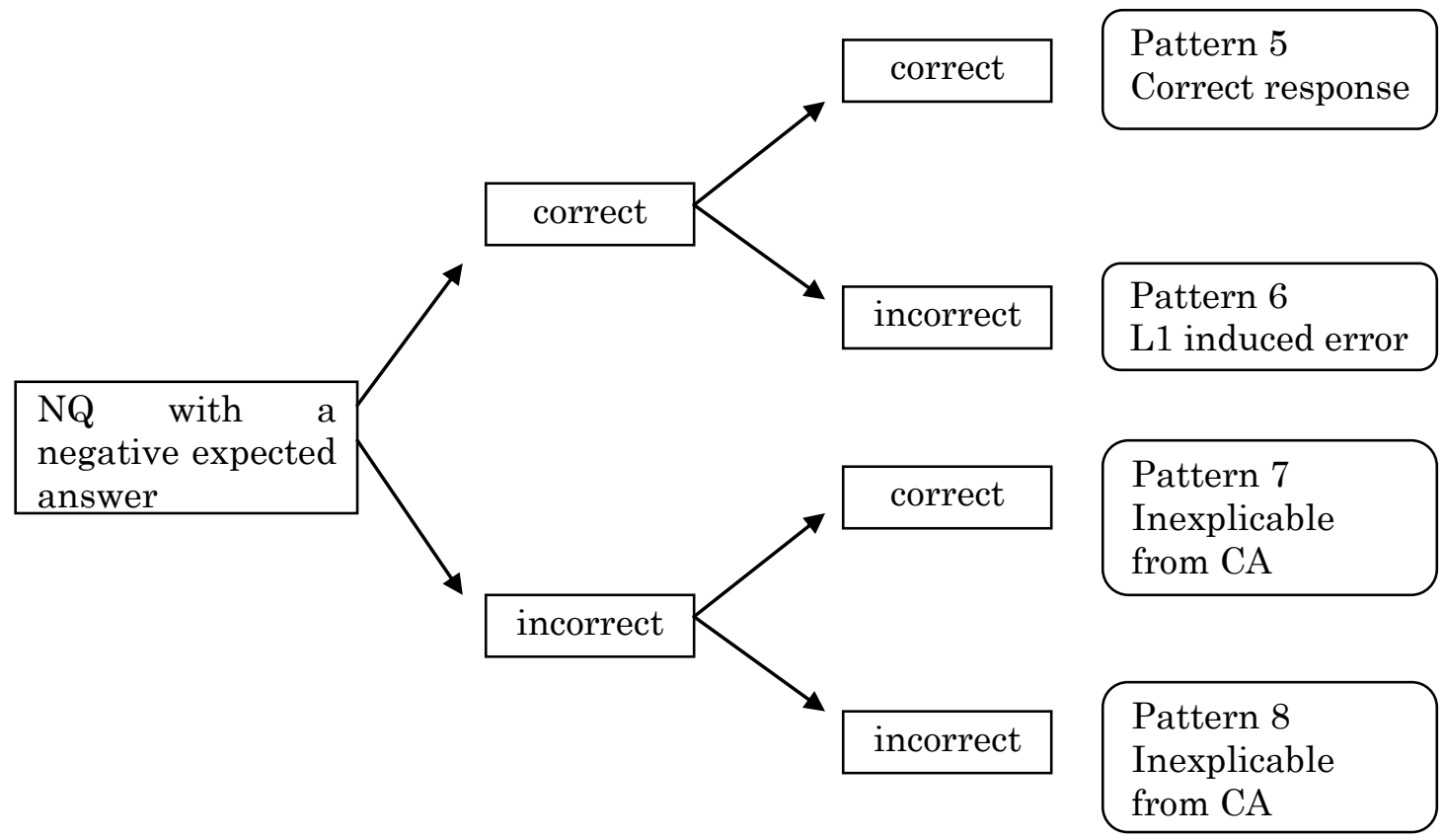




\section{Empirical Studies of the Acquisition of Negative Questions}

This section presents empirical studies that discuss the difficulties Japanese learners of English have in acquiring English NQs. The findings from these studies are compared with the prediction presented above, and the approach for the current research is explained.

Takashima (1989) investigates how Japanese EFL learners respond to English negative interrogatives in relation to $\mathrm{L} 1$ influence. A questionnaire containing 2 negative questions was administered to 288 university students in his study. The questions were given without any context, and the students were told simply to choose either yes or no to each question. Some of the participants had an oral interview after the data were collected. Following are the stimulus sentences used in the study:
a. Do you like English?
Yes No
b. You don't like English?
Yes No
c. Don't you like English?
Yes No

The answers were judged based on their consistency - i.e. a student who liked English would answer "yes" to all the questions, and a student who did not like English would answer "no." The results show that those who gave "prescriptively" correct answers, choosing either yes or no for all the questions, accounted for just $24 \%$ of the total participants. The other participants' responses show variations in their $\mathrm{Y} / \mathrm{N}$ response among the three questions. The results suggest that Japanese learners of English have a lot of trouble when responding to English NQs.

A noticeable tendency observed in the participants' answers is that they are more likely to give an incorrect response in question type 12(b) rather than 12(c). The data show that just $9 \%$ of the participants chose a correct response only in 12(b), but 
the percentage increased up to $38 \%$ for those choosing a correct answer only in 12 (c).

Takashima attributes this tendency to the Japanese EFL curriculum, which usually does not deal with a descriptive use of English like 12(b) in detail. Having oral interviews with some of the participants, Takashima found that all the subjects he interviewed remembered explicitly taught grammar and applied it when answering the questionnaire. The relatively low performance for 12(b) is explicable by the fact English teachers in Japan rarely deal with its structure. From this study, Takashima draws the conclusion that L1 does influence learner's interlanguage construction with respect to negative interrogatives and implies that explicitly learned knowledge can help learners choose the correct response.

The questionnaire used in Takashima's (1989) study is not designed to elicit learners' spontaneous performance. Although it has the advantage of collecting a large number of data with ease, the research design overlooks an important component of NQs, i.e., context. In his study, the two NQs were presented without context, and the content of the questions is identical. That is, there were some participants who answered yes to 12(a) and were then asked 12(b), which only makes sense where the questioner holds a negative assumption about the proposition. Questionnaires of this type are awkward even in Japanese, and it is quite possible that the questions induced participants to rely on the taught rule.

Another important issue that is not addressed in Takashima's study is the underlying intention of the $\mathrm{Y} / \mathrm{N}$ response. Although Takashima interpreted the underlying intention for the two NQs based on the $\mathrm{Y} / \mathrm{N}$ response to 13(a) it can be assumed that some participants might have changed their opinions during the test. 
Suppose you are taking the questionnaire and put no to the first two questions. The sequence is reasonable because the second question can be interpreted as confirmation seeking. When it comes to the third question, due to the questioner's relentless inquiry, it seems more natural to assume that the questioner expects you to say that you like English. That is, some participants may have conveyed their intended meaning accurately for all three questions even if their responses do not match what Takashima considers to be correct.

Since no measure was taken in Takashima's study for pinpointing the participant's interpretation of the stimulus sentences, it is not reasonable to draw a firm conclusion based on the data. However, the results of his study suggest that Japanese learners of English have a proclivity to interpret the polarity of the expected answer as negative, the only case where the Japanese $\mathrm{Y} / \mathrm{N}$ response conflict with English. Although two interpretations are possible with respect to the polarity of the expected answer, most of the subjects gave responses which comply with the Japanese default answering pattern. Even with the possibility of some participants' changing their opinion, the high percentage of the participants who did not give correct responses supports the hypothesis that the L1 negatively influence the TL production.

Kang and Lim-Chang (1998) examined the effects of TL exposure on the acquisition of negative questions. The participants in their study consisted of two language groups: Korean and Japanese. The Korean group included 10 English Language Institute (ELI ) students, 15 graduate students (their major not indicated), 10 housewives, and 10 children who attend a Korean school in Florida. The Japanese group consisted of 4 ELI students and 4 graduate students. These participants were 
given an oral interview including five negative interrogatives along with some distracters. Following are four of the five NQs given to adults subjects in the study (the other question is not listed in the article):

(13) a. Aren't you a student back in your country? (for ELI students) Aren't you a graduate student? (for graduate students, housewives)

b. Didn't you learn English before you came here?

c. English is not easy, is it?

d. Don't you speak English at home?

Limiting the discussion to the case of Japanese subjects, the results show that there was a large difference between subjects who had stayed in US less than two years and those more than 2 years; whereas the accuracy rate of the former was $66.7 \%$, that of the latter reached more than $90 \%$. The result, though limited in the number of subjects, was interpreted as showing a relationship between length of stay and accuracy of responses to negative questions in English. The detailed results of the participants' performance are not presented in the study.

The oral interview used in Kang and Lim-Chang (1998) is problematic. Although it has the advantage of eliciting from every participant responses to the same stimulus sentence, it is highly likely that the NQs were asked without an appropriate context (Nagao, 2005). For example, in a natural context question 14(d) would require prior discourse in which interviewees provide details about what they do at home. Although an interview is more likely to elicit responses that reflect the participants' spontaneous performance, attention must still be paid to the context of NQs.

Nagao (2005) included investigation of how Japanese learners of English choose $\mathrm{Y} / \mathrm{N}$ responses to negative interrogatives in comparison to native English speakers. The study consisted of 22 native English speakers and 22 Japanese students. 
The Japanese subjects were 8 undergraduate students and 14 graduate students, and the mean length of stay in an English-speaking environment of those students was 3 years and 7 months. The subjects were shown 21 short video clips containing different types of negative questions. After each clip, the subjects were asked to choose either yes or no in the given situation. The proposition of the $\mathrm{Y} / \mathrm{N}$ response was presented, so the participants only needed to choose a $\mathrm{Y} / \mathrm{N}$ response that fit in the context. The stimulus sentences included three different types of syntactic structure along with differences in the polarity of expected answer, intonation, and pragmatic functions. The syntactic structures included interrogative, declarative with tag and declarative.

The comparison of the two groups' performances indicates that the Japanese students had a higher rate of accuracy with respect to the practiced rule than the American group did. Nagao attributes this unexpectedly high accuracy of Japanese students to the influence of explicitly taught knowledge of English. Because $\mathrm{Y} / \mathrm{N}$ responses were followed by the addressee's proposition, many of the Japanese subjects were likely to have chosen their answer in accordance with the polarity of the proposition.

Nevertheless, some of the Japanese subjects showed variation in their response patterns. Nagao argues that the variation cannot be explained simply by L1 transfer for several reasons. The interview with the Japanese subjects, for example, revealed that they consciously chose answers that departed from the taught rule when they felt it more natural to do so. Those subjects had a certain amount of TL exposure, so it seems likely that they had experienced miscommunication with native speakers, which eventually helped modify their interlanguage. The fact that most of the Japanese 
subjects' variation was seen where American subjects also varied supports this analysis. Nagao's research indicates that with increasing length of TL exposure, learners' response pattern approaches the native speakers' norm.

The research design of Nagao (2005) also has several deficits with regard to eliciting an authentic performance. First of all, Nagao did not include any distracters in his research, which probably drew the participants' attention to NQs. It seems likely that learners will resort to their learned knowledge when they realize they are being tested. The fact every question prompted $\mathrm{Y} / \mathrm{N}$ responses in accordance with the proposition might have been an artifact of the participants recognizing the focus of the study and applying their learned knowledge.

There is another problem in the stimulus sentences used in his study. Although it has the advantage of eliciting responses to various types of NQs, it is possible that the subjects would not give $\mathrm{Y} / \mathrm{N}$ responses to every different kind of $\mathrm{NQ}$. The NQs whose structure are declarative, for example, are not dealt with in Japanese EFL, so the Japanese subjects may not give $\mathrm{Y} / \mathrm{N}$ responses to those questions in practice. Again, the subjects might have provided answers to the NQs simply by applying the taught knowledge.

It should be noted, however, that his research study is quite solid in the sense that great care was taken to present authentic usage of NQs. The stimulus sentences were excerpts from several popular sitcoms. Each stimulus sentence was given to participants only after they were shown the prior context, so the participants should have understood the rationale behind the NQ usage.

In order to elicit data that reflects the spontaneous performance of Japanese 
learners of English, a research method requires conscious manipulation of stimulus sentences and elicitation of $\mathrm{Y} / \mathrm{N}$ responses. The research studies presented above all have their own strengths in data collection, but their instruments also introduced certain possible confounding factors. Takashima (1989) and Nagao (2005) draw the participants' awareness towards $\mathrm{Y} / \mathrm{N}$ responses by asking them NQs without sufficient distracters. The oral interview of Kang \& Lim-Chang (1998) is effective in avoiding raising awareness of NQs, but the method lacked considerations of the context behind asking NQs. The present study, which attempts to investigate Japanese students' spontaneous response patterns, is designed to eliminate possible confounding influences on learners' natural performance.

\section{Research Questions}

The present study investigates the knowledge source Japanese learners of English use when responding to negative interrogatives in English. Because transfer-based learning theory is the most common and popular explanation for the misuse of $\mathrm{Y} / \mathrm{N}$ response, this study is designed to analyze the Japanese learners' performance in relation to their L1. The theoretical assumption behind this study is that if L1 plays a role in the acquisition of negative interrogatives, learners will show variation in their response patterns where the $\mathrm{L} 1$ allows for deviation from the default response pattern, i.e. yes is followed by a negative tag and no by a positive tag.

The degree of L1 influence in NQ responses is still an open question. There has been no research that attempted to describe how the L1 interferes with or sometimes facilitate the acquisition of NQs, because analyzing the extent to which L1 
influences the acquisition is a quite complicated issue. The previous literature implies that there are two possible learning pitfalls, interpretation of the stimulus sentence and the choice of the $\mathrm{Y} / \mathrm{N}$ response. Researchers who seek to investigate the picture of interlanguage construction need to give consideration to the two pitfalls and their interaction. Hoping to contribute to this area of interlanguage analysis, this study addresses the following two research questions.

1. How do Japanese learners of English choose yes or no in responding to English negative interrogatives? In particular, do they appear to follow more of an English-system norm or a Japanese-system norm?

2. How do Japanese learners of English interpret the polarity of the expected answer of English NQs? In particular, do they appear to follow more of an English-system norm or a Japanese-system norm? 


\section{Chapter 3: Research methodology}

This chapter presents the methods used in the present research study as well as the rationale behind the methodology. First, the demographic information of the participants is presented. Second, the data collection instruments and the procedure established through pilot studies are presented. At the end of this chapter, the data analysis procedures are presented.

\section{Participants}

This study involved 8 Japanese learners of English, 4 females and 4 males, attending Portland State University (PSU). In order to elicit data that reflect the effect of Japanese English Language Teaching (ELT), the subjects were chosen so that at the time of data elicitation, they had less than 6 months of experience in an environment where English was a primary communicative tool. In addition, all the participants had English instruction in Japan at least through high school.

The demographic information of the participants is summarized in the Table 2. The age of the participants ranged from 19 to 22 , and the length of stay in the target language (TL) environment was 4 to 5 months. TOEFL scores, an indicator of English proficiency, ranged from 500 to 567 , and the average score was 539, which most universities in the United States consider sufficient to move into introductory work as undergraduate students in English. The participants in the present study had about the same English proficiency except that one participant, Shun, had a relatively low proficiency compared to the other participants. 
Table 2: The demographic information of the participants

\begin{tabular}{|c|c|c|c|c|}
\hline $\begin{array}{c}\text { Name } \\
\text { Pseudonym) }\end{array}$ & Age & Gender & Highest TOEFL score & $\begin{array}{c}\text { Length of stay in an } \\
\text { English speaking } \\
\text { environment }\end{array}$ \\
\hline Ai & 22 & Female & $72(\mathrm{iBT})(=533$ in PBT) & 4 months \\
\hline Hana & 21 & Female & $73(\mathrm{iBT})(=533$ in PBT) & 5 months \\
\hline Naoko & 21 & Female & $567(\mathrm{PBT})$ & 4 months \\
\hline Yuri & 19 & Female & $547(\mathrm{PBT})$ & 4 months \\
\hline Keita & 20 & Male & $567(\mathrm{PBT})$ & 4 months \\
\hline Shun & 21 & Male & $500(\mathrm{PBT})$ & 4 months \\
\hline Yoshiki & 21 & Male & $533(\mathrm{PBT})$ & 4 months \\
\hline Yuta & 21 & Male & $74(\mathrm{iBT})(=537$ in PBT $)$ & \\
\hline
\end{tabular}

(iBT: internet-based test, PBT: paper-based test)

The study was approved by the Human Subjects Research Review Committee and participants signed consent forms before data collection began.

\section{Pilot studies}

As a preliminary step, four pilot studies were conducted in order to refine the procedures for the research. The first two pilot studies were conducted to establish a basic data collection method. The other two pilots were held to refine the research design. Since, to the best of my knowledge, there was no previous research study that attempted to analyze the process of the question and answer sequences in detail, the present research methodology required a great deal of effort to refine procedures and eliminate variables that could affect the results. The following sections present the 
final research methodology of the present study established through the pilot studies.

\section{Data Collection instruments}

Because this study aims at eliciting the spontaneous response pattern of Japanese learners of English and analyzing learner processes in detail, it used two data collection methods. The first data collection method was an oral interview with a native English speaker, and the second was a retrospective protocol analysis of the interview. The interview was designed to investigate the participants' spontaneous response pattern, which eventually served to address the first research question. The second research question, which examined the internal process participants used to reach their response patterns, was addressed through the retrospective protocol analysis. In what follows, the description of the two data collection methods is presented.

\section{Oral interview}

The first data collection method was an oral interview conducted by a native English speaker. Each participant was interviewed individually. The time allotted to the interview was about 10 minutes. From the pilot studies, it was discovered that 10 minutes was sufficient to obtain 12 responses to NQs as well as establishing a sound context for each stimulus sentence. Twelve responses were determined to be an appropriate target because in the pilot studies 12 responses produced clear patterns in learner responses.

The interview was highly structured with the interview questions prepared in advance through pilot studies. The stimulus sentences, which will be presented in the 
following section, required a context to be appropriately used, so an interview scenario was also prepared. The stimulus sentences and a rough script of the way to establish the context were given to the interviewer to use during the interview (Appendix).

Efforts were made to avoid calling attention to NQs. The participants were informed prior to the interview that this research study was aimed at improving the quality of Japanese EFL. The interview topic was classes at PSU and how they compared with ones in Japan, a topic that was quite natural for students to discuss. The interview itself used not only NQs but also positive questions, and the interviewer also made some attempts to draw attention away from the NQs by talking about some irrelevant topics or expressing his opinions from time to time. The interviewer did not ask for clarification of any $\mathrm{Y} / \mathrm{N}$ responses and kept the conversation flow natural. The naturalness of the interview scenario was increased through the pilot studies because the interviewer had practiced interviewing several times before the real study began. Only one of the subjects reported suspecting the research purpose of the interview, which suggests the interview seemed natural to most of the participants.

The interviews were videotaped. The videotapes were used in analyzing the interaction and in the retrospective protocol analysis.

\section{Stimulus sentences}

This study was focused on NQs used for information seeking. The total number of NQs used in this study was 12 . For the sake of simplicity, the syntactic structure of the stimulus sentences is restricted to interrogative, i.e. tag questions and declaratives functioning as questions are excluded. The stimulus sentences were 
divided into two types based on the polarity of the expected answer: i.e., ones with a positive expected answer and ones with a negative expected answer. An identical number of the stimulus sentences was prepared for each type of NQ.

The stimulus sentences were based on the topic of classes the participants had taken in the previous term and how they compared with English classes in Japan. This speech topic was chosen since many exchange students are familiar with it. Having a speech topic that the participants feel comfortable talking about enhances smooth communication, which then helps to establish the context and to ask NQs based on it. Table 3 presents the stimulus sentences used in this study.

Table 3: Stimulus sentences

\begin{tabular}{|c|c|c|}
\hline \# & Stimulus sentence & Polarity of the expected answer \\
\hline 1 & Don't you think that taking \# classes is too much? & Positive \\
\hline 2 & Didn't you take ...? & Negative \\
\hline 3 & Wasn't that difficult for you? & Negative \\
\hline 4 & Didn't you have ... in the class? & Negative \\
\hline 5 & Wouldn't it have been better if you had more ...? & Positive \\
\hline 6 & $\begin{array}{c}\text { Isn't it nice to have discussion and exchange } \\
\text { opinions? }\end{array}$ & Positive \\
\hline 7 & Wasn't it hard for you to take classes in English? & Positive \\
\hline 8 & Didn’t you study ...? & Negative \\
\hline 9 & $\begin{array}{c}\text { Wouldn't it have been better if the teacher used } \\
\text { English (Japanese) in the class? }\end{array}$ & Positive \\
\hline 10 & $\begin{array}{l}\text { Don't you hope that in the future, Japanese schools } \\
\text { focus more on conversation? }\end{array}$ & Positive \\
\hline$*(10)$ & Don't you agree with him? & Negative \\
\hline 11 & Don’t you like ...? & Negative \\
\hline 12 & Didn't Kosuke tell you to bring it? & Negative \\
\hline$*(12)$ & Wasn't this interview easy for you? & Positive \\
\hline
\end{tabular}

*The numbers in parenthesis are to be asked to meet the circumstances of the different 
interviews. That is, the interviewer picked the appropriate question based on how the interview developed up to that point.

The NQs were divided into two types: those with a positive expected answer and those with a negative expected answer. All the NQs were preceded by a prior discourse, which established the polarity of the expected answer. For example, question number 2 had prior discourse where the interviewer asked a question about classes the participant took in the previous term. The specific class used in this stimulus sentence was decided based on the context. That is, if a participant answered that she took Business, Economics, and P.E., question 2 might be "didn't you take an English class?" This stimulus sentence was treated as a NQ with a negative expected answer because the prior discourse caused the interviewer to hold a negative assumption about the question.

Stimulus sentences 1 and 7 did not have prior discourse that specified the polarity of the expected answer. However, these questions were categorized as having a positive expected answer on a natural assumption that it is difficult to do university-level study in a foreign language.

\section{Retrospective protocol analysis}

After the interview, all the participants participated in an individual retrospective analysis interview. In this session, I was the interviewer and the interviews were conducted in Japanese. In order to address research question 2, three interview questions were prepared regarding the stimulus sentences and the intention of their responses. At the end of this session, the participants were asked a few 
questions related to their previous experience with English NQs.

Before the retrospective protocol analysis of the interview, the participants were informed that the research was intended to look into how Japanese learners of English respond to NQs. Although some attempts had been made in the pilot studies to mask the research purpose throughout the retrospective protocol analysis, it turned out to be ineffective because it was quite difficult to ask questions about NQs without raising awareness about the difference between the two languages. Revealing the research purpose at the beginning actually facilitated the retrospective analysis.

Three interview questions for each stimulus sentence were prepared for the retrospective protocol analysis. The first question concerned the comprehension of the context. This question was prepared because the context behind the NQ usage was one of the crucial factors that dictated the expected answer. Furthermore, the pilot studies showed several cases where participants answered NQs without comprehending the prior discourse. Properly understanding the process that participants used to answer a NQ required knowing how they understood the process.

The second interview question concerned comprehension of the stimulus sentences. This question was addressed in two ways. First, the participants were orally asked whether they had understood the syntactic structure of the stimulus sentence correctly. This question was prepared because there were several cases in the pilot studies where a participant responded to NQs without understanding the syntactic structure of the stimulus sentence. For example, in one of the pilot studies, there was a learner who misunderstood a NQ as a positive question. It is highly possible that misunderstanding the syntactic structure of the stimulus sentences is one of the covert 
errors that are involved in interactions with NQs. In the analysis, minor errors, such as particles and plural $-\mathrm{s}$, were not taken into account.

Secondly, the participants were given a translation task for all the stimulus sentences. In the translation task, the participants were given four translation patterns for the stimulus sentence and asked to choose one option that best described their interpretations. For example, following are the four translation patterns for a stimulus sentence "Wasn't that difficult for you?":

(14) a. sono jugyou-wa muzukashiku nakatta no desuka?

that class difficult be-not that be

b. sono jugyou-wa muzukashiku nakatta no? that class difficult be-not that

c. sono jugyou-wa muzukashikatta $\mathrm{n}$ janai? that class difficult that be-not

d. sono jugyou-wa muzukashikatta no dewa arimasenka? that class difficult that be-not

The first two translations, 14(a) and 14(b), are the ones with a negative proposition, i.e. the interpretation of these sentence is closer to "Is it the case that the class was not difficult?" Translations 14(c) and 14(d) have a positive expected answer, i.e. "Isn't it the case that the class was difficult?" The difference between 14(a) and 14(b) as well as between $14(\mathrm{c})$ and $14(\mathrm{~d})$ is the degree of politeness. Stimulus sentences 14(a) and 14(d) have the polite ending morpheme -desu and -masen respectively, whereas $14(\mathrm{~b})$ and $14(\mathrm{c})$ don't. The distinction is intended to help the participants find the best translation equivalent. The order of the options was randomized question by question so that a participant would not choose one option automatically.

The intention of having those four translation patterns was to elicit how the 
participant interpreted the polarity of the expected answer. As described in Chapter 2, the translation options (a) and (b) are the default interpretation of NQs in Japanese, and this type of NQ elicits Y/N responses that do not parallel to the English norm. The translation options (c) and (d), on the contrary, elicit the $\mathrm{Y} / \mathrm{N}$ response consistent with the English counterparts, for these questions contain a positive proposition. Having a participant choose from these translation options revealed their interpretations of the polarity of the expected answer, which served to reveal the process they used to respond to a NQ.

The final step in the retrospective analysis was to ask participants their intention behind any simple "yes" or "no" answers. Since no attempts were made during the interview to clarify the intended meaning of $\mathrm{Y} / \mathrm{N}$ responses, this question was necessary to determine whether any miscommunication had happened or not.

\section{Data Collection Procedure}

Interviews were conducted individually with each participant. Each interview was held at a study room in the PSU library. After submitting a consent form, the participant was invited into the room where the videorecorder was set up. After the interviewer and participant introduced themselves, a general overview of the interview was explained to the participant. The videorecording started when these procedures were finished, and the interview was conducted. At the end of the interview, the participants were given a questionnaire in which they filled in their personal information such as their TOEFL scores and length of stay in the TL environment. The interviews were held at each participant's convenience and were spread over three 
days.

The retrospective protocol analysis session was also held at each participant's convenience. Ideally, the session was held soon after the interview so that the participants could recall their performance in detail, but the actual time delay ranged from four to eight days. Prior to the retrospective analysis, I prepared four translation patterns for each stimulus sentence based on the particular interview discourse for that participant. Since each stimulus sentence was given in a context specific to the individual interview, the translation task required modifications from participant to participant.

The retrospective protocol analysis session started with an inquiry about whether the participant had found the interview peculiar in any way. This question was intended to make sure their performance was spontaneous with respect to answering NQs. Then, the instructions for the retrospective protocol analysis were given. The instructions asked participants to describe the way they had understood and responded to the stimulus sentences at the time of the interview.

After the instructions were given, the video clip was played. Every time a stimulus sentence was given, the video clip was paused, and the participants were asked the three interview questions concerning their comprehension of the context, their comprehension of the stimulus sentence, and their translation choice. This procedure was carried out for all the stimulus sentences.

At the end of the interview, I asked the participants three additional questions: the strategy they use in responding to NQs, the way they were taught English NQs, and their experience with NQs. These questions were intended to consider possible 
factors that influenced the way the participants responded to English NQs and pedagogical implications for Japanese EFL.

\section{Data Analysis Procedures}

The results of the NQ interview were analyzed by calculating the number of errors in responses to NQs, with separate categories for NQs with a positive expected answer and a negative expected answer. To begin the analysis, I reconsidered the polarity of the expected answer for each stimulus sentence because the actual interviews sometimes developed in unexpected ways. The polarity of the expected answer is quite sensitive to the prior discourse, and the parts of the interview that failed to establish the context as planned were reanalyzed. The polarity of the expected answer of all the stimulus sentences was confirmed with the interviewer.

When this process was complete, each participant's performance was analyzed for each stimulus sentence. The intention of each $\mathrm{Y} / \mathrm{N}$ response was confirmed through the retrospective protocol analysis, and the percentage of correct responses was calculated for each category of NQ. Patterns in the responses were analyzed for the group overall and for each individual.

The results of the retrospective protocol analysis were analyzed by first comparing the interpreted polarity of the expected answer with the correct polarity. As described in the Data Collection Instrument section, the Japanese translations contain a proposition of either polarity. The comparison between the two was aimed to investigate whether the participants had correctly understood the polarity of the expected answer. The results of this analysis were then investigated along with the 
participants' comprehension of the context and the syntactic structure of the stimulus sentence.

Finally, the results of all the data analysis were synthesized in order to answer the research questions. The analysis of their $\mathrm{Y} / \mathrm{N}$ response patterns was to answer the first research question. I looked into the participants' response patterns based on stimulus sentences, which served to address whether there is a relationship between their interlanguage and Japanese. I compared their $\mathrm{Y} / \mathrm{N}$ response patterns with what a Japanese Y/N response would produce, so that I could suggest whether L1 influence their performance.

The retrospective analysis of the interview was used to answer the second research question. This analysis reveals how Japanese learners of English interpret English NQs. I investigated whether there were any trends in how the participants interpreted English NQs. 


\section{Chapter 4: Results and Discussion}

This chapter reports and interprets the results of the interview and retrospective protocol analysis. First, the results of the interview are presented. In the second section, the results of the retrospective analysis are presented. Then, in the discussion section, detailed analysis of the errors observed in this study is presented with some considerations of a few external variables. At the end of the chapter, answers to the two research questions of the study are summarized.

\section{Results of the interview}

The number of negative questions (NQs) given in the interviews was 94. Fifty NQs had a positive expected answer, and 44 had a negative expected answer. The total number of the stimulus sentences was originally set to be 96 (12 NQs for 8 participants), but in the actual interview, there was one case where the context did not allow for asking a NQ and another case where the interviewer simply forgot to ask the question.

Most participants received the same stimulus sentences. However, for stimulus sentence 10 (see Table 1 in Chapter 3) discussion with one participant, Yuta, did not allow the same stimulus sentences to be given. Thus, the difference in the number of the stimulus sentences between NQs with a positive expected answer and ones with a negative expected answer was attributable to the failure to establish a negative assumption on the proposition in question.

Table 4 describes the general results of the interview. All the tokens of NQs are categorized as having either a positive expected answer or a negative expected answer. 
The second column of table 4 shows the number of NQs given to the participants. The third column presents the number of times that "yes" or "no" was not used in the response, and the fourth shows the number of misused $\mathrm{Y} / \mathrm{N}$ responses.

Table 4: The results of the interview

\begin{tabular}{|c|c|c|c|}
\hline & $\begin{array}{c}\text { Number of } \\
\text { stimulus sentences }\end{array}$ & $\begin{array}{c}\text { Number of times } \\
\text { where a Y/N was } \\
\text { not elicited. }\end{array}$ & $\begin{array}{c}\text { Number of misused } \\
\text { Y/N responses }\end{array}$ \\
\hline $\begin{array}{c}\text { NQs with a } \\
\text { positive expected } \\
\text { answer }\end{array}$ & 50 & 15 & 0 \\
\hline $\begin{array}{c}\text { NQs with a } \\
\text { negative expected } \\
\text { answer }\end{array}$ & 44 & 14 & 3 \\
\hline Total & 94 & 29 & 3 \\
\hline
\end{tabular}

The total number of NQs that did not elicit yes or no was 29. In many of these cases, the participants stated just a proposition (e.g. saying 'I (don't) think so') or gave an elaborated response of their opinion. Some participants said in the retrospective protocol analysis that some of the questions were not easily answered in a clear-cut yes/no way. This verbal behavior may be the participants' deliberate strategy to avoid $\mathrm{Y} / \mathrm{N}$ responses or may simply be their spontaneous response pattern to make communication clear. In this study, however, this question will not be addressed since the research was not designed to address this issue.

The number of incorrect $\mathrm{Y} / \mathrm{N}$ responses observed in the interview was only 3, which accounts for approximately $3 \%$ of the total NQs. The number of participants who made the incorrect $\mathrm{Y} / \mathrm{N}$ responses was two, Ai and Yuri. Two errors were observed in Ai's interview and one in Yuri's. The results show that, contrary to the common belief that Japanese learners of English tend to make errors in $\mathrm{Y} / \mathrm{N}$ responses 
to NQs, the participants in this study rarely made an incorrect response.

The error analysis of the $\mathrm{Y} / \mathrm{N}$ responses reveals that the errors occurred only when the stimulus sentence held a negative expected answer. As described in the previous chapters, the Japanese response system causes a $\mathrm{Y} / \mathrm{N}$ response inconsistent with English only when a question contains a negative proposition. This finding leads one to ponder the relationship between the participants' behavior and Japanese.

Looking into the errors, I found that the response patterns are characteristic of Japanese $\mathrm{Y} / \mathrm{N}$ responses. As described in Chapter 2, the English response system allows for variation in $\mathrm{Y} / \mathrm{N}$ responses (Nagao, 2005). When a NQ holds a negative expected answer, the response pattern allows for three answers: 'Yes' followed by a positive tag, 'no' followed by a negative tag, and 'no' followed by a positive tag. Comparing the results of this study with these descriptively correct responses, I found that none of the three tokens fell under any of the patterns acceptable in English. Following is one of the errors observed in Ai's interview (S stands for "Student" in all the interviews, and I for "Interviewer"):

1) I: What were the classes (you took last term)?

S: One is English, one is sustainability, and African history.

I: That sounds interesting. So, didn't you take any IELP classes?

S: Yeah.

In response to the stimulus sentence, Ai answered yeah meaning she did not take an IELP class. The two other misuses of the $\mathrm{Y} / \mathrm{N}$ response were also the same pattern, i.e., yes intended as an agreement to a negative proposition. The fact that this response pattern is grammatical in Japanese but not in English suggests that the L1 interfered with the production of a $\mathrm{Y} / \mathrm{N}$ response in those three cases. 
The results of the interview show that the participants did not make incorrect Y/N responses as often as reported in previous studies. Furthermore, for those NQs with a positive expected answer, none of the participants gave an incorrect response. The question arises why, contrary to the common understanding, the participants rarely made incorrect responses. The results of the retrospective protocol analysis help us better understand the process by which the participants reached their response.

\section{Results of the retrospective protocol analysis}

The retrospective analysis started by asking the participants if they thought the interview was strange in any way. Even with the interview instruction and its content, there was one participant, Naoko, who realized that the interviewer tried to elicit responses to NQs. All of the other participants thought that the interview was to better understand international students from Japan. Therefore, most of the interview data can be considered their unmonitored pattern for responding to NQs. The results of the retrospective protocol analysis show that there are many covert errors involved in the question and answer sequence of NQs. Table 5 presents the overall summary of the retrospective analysis. 
Table 5: The general results of the retrospective protocol analysis

\begin{tabular}{|c|c|c|c|}
\hline & $\begin{array}{c}\text { \# of times the } \\
\text { subjects did not } \\
\text { understand the } \\
\text { context correctly }\end{array}$ & $\begin{array}{c}\text { \# of times the } \\
\text { participant did not } \\
\text { correctly understand } \\
\text { the syntactic } \\
\text { structure of stimulus } \\
\text { sentences }\end{array}$ & $\begin{array}{c}\text { \# of times the } \\
\text { participant } \\
\text { misinterpreted the } \\
\text { polarity of the } \\
\text { expected answer }\end{array}$ \\
\hline $\begin{array}{c}\text { NQs with a } \\
\text { positive expected } \\
\text { answer } \\
(\mathrm{N}=50)\end{array}$ & 2 & 29 & 6 \\
\hline $\begin{array}{c}\text { NQs with a } \\
\text { negative } \\
\text { expected answer } \\
\text { (N=44) }\end{array}$ & 1 & 6 & 0 \\
\hline $\begin{array}{c}\text { Total } \\
\text { netal }\end{array}$ & 3 & 35 & 6 \\
\hline
\end{tabular}

The speech topic was quite familiar to the participants, and there were only 3 cases where the participants did not understand the context of the stimulus sentence. Two cases were observed in Yoshiki's interview, and the other case in Naoko's. The following dialogue is the transcript of Naoko's interview where the context was not understood:

2) I: Do you think that English classes you took in Japan helped you with your conversation skills?

S: No, I don't think so.

I: Okay. I know that Kosuke thinks Japanese schools aren't very good at teaching communication skills. So, don't you hope that in the future, Japanese school focuses more on teaching conversation and communication?

S: Yes.

In the retrospective analysis, Naoko explained that she misunderstood the interviewer's statement preceding the stimulus sentence as "Kosuke thinks Japanese schools ARE very good at teaching communication skills." According to her analysis, 
the contradiction between her interpretation of the preceding statement and her opinion about the proposition confused her, so she could not understand the stimulus sentence. In this interaction, she understood the stimulus sentence as a statement "In the future, Japanese schools focuses more on teaching conversation and communication." Her response, yes, was not a $\mathrm{Y} / \mathrm{N}$ response but a backchannel intended to function as acknowledgment.

In all of the three cases where the participants did not understand the context, the participants misunderstood the stimulus sentences. Two cases show that the participants took the stimulus sentence as a statement, and in the other case, the participant did not properly understand the stimulus sentence but guessed correctly. Just as in any verbal interaction, the proper understanding of the context and stimulus sentence is vital in appropriate communication. Especially an interaction with NQs requires the proper understanding of the context because it can trigger a deviation from descriptively correct rules. It is necessary to understand the context correctly to properly respond to NQs.

Inquiry on whether the participants properly understood the stimulus sentence elicited rather unexpected results. Of the total 94 questions, the participants did not correctly understand 35 (37\%). The average number of misunderstandings per participant was 2.9, and the number ranged from 2 to 12 . In particular, many of the participants expressed their confusion towards two of the stimulus sentences. Following are the two stimulus sentences:

3) Wouldn't it have been better if you had ...?

4) Don't you hope that Japanese schools focus more on teaching conversation in the future? 
Comparing these two stimulus sentences with the others, one finds that these two stimulus sentences are more complex or longer. Stimulus sentence (3) uses the subjunctive mood, which a lot of Japanese learners of English have trouble with, and (4) contains a lengthy complement clause compared with other stimulus sentences.

In most of the cases where they did not correctly understand the syntactic structure, the participants took the stimulus sentences as a positive question. In fact, the number of the stimulus sentences interpreted as a positive question amount to 30 , which accounts for the majority of errors in understanding the syntactic structure. This finding indicates that the negative morpheme in the stimulus sentence does not draw as much attention as content words in the stimulus sentence do. In fact, one of the participants, Shun, did not recognize the existence of the negative morpheme for any of the 12 stimulus sentences he received. Because responses to a positive question do not conflict between Japanese and English, his $\mathrm{Y} / \mathrm{N}$ responses did not cause any miscommunication.

As participants explained their comprehension of the difficult stimulus sentences, it appeared that they understood only the general contents without paying attention to details. For example, none of the participants could process the syntactic structure of stimulus sentence (3). The participants explained that they could respond $\mathrm{Y} / \mathrm{N}$ to this question because they understood the latter half of the stimulus sentence, "better if you had more lecture?" They assumed the general meaning of the stimulus sentence out of this clue and made an appropriate response.

The stimulus sentence in (4) elicited a different type of misunderstanding of the syntactic form. This question was given to seven of the total participants, and four 
out of the seven participants understood it as a statement. Intriguingly, three of the participants gave a response of yes or yeah to this stimulus sentence. They explained that the yes/yeah was only a backchannel functioning as acknowledgement. That is, they understood only the content of the subordinate clause "Japanese schools focus more on teaching conversation in the future," and gave a backchannel to this statement. This response did not cause miscommunication since their opinion about the proposition in question coincided with the backchannel "yeah;" in other interactions, it could confuse a questioner.

The results of the translation task showed that there were several cases where the participants misinterpreted the polarity of the stimulus sentences $\left(4^{\text {th }}\right.$ column in table 4). The total number of the misinterpretation was 6 , and all the tokens were observed in stimulus sentences that have a positive proposition. The errors were made by 5 of the participants, and all five misinterpreted the polarity of the proposition for the same stimulus sentence. Following is the stimulus sentence with its context (from Ai's interview):

5) I: Was last term your first term here at PSU?

S: Yes, it was my first term.

I: Wasn't it hard for you to take classes in English?

S: Yeah, of course.

In this dialogue, $\mathrm{Ai}$ interpreted the stimulus sentence as having a negative proposition (i.e., as though the interviewer said "it was not hard for you to take classes in English, was it?"). Four other participants similarly misinterpreted the polarity of the proposition in this question. 
The other error was observed in the following stimulus sentence (from Yuri's interview):

6) I: Do you like discussions?

S: If I can speak English well, I like it.

I: I heard that many Japanese don't like discussions, but isn't it nice to have discussions and exchange opinions with other students?

S: Yeah.

Yuri misinterpreted the polarity of the expected answer for the stimulus sentence in (6): She interpreted the stimulus sentence as "it is not nice to have discussions and exchange opinions with other students, is it?" In both of the cases in (5) and (6), the stimulus sentences were given to the participants with a positive expected answer, but some of the participants' interpretation turned out to be the opposite of the interviewer's intention. In the discussion section, a possible source of the error will be discussed.

The results of the retrospective protocol analysis revealed that there are more errors involved in the interaction with NQs than what is apparent on the surface. Retrospective protocol analysis showed that there are basically two covert error types. The first concerns the error of misunderstanding the syntactic structure of the stimulus sentence. Inquiry into their comprehension of the stimulus sentences showed that more than one-third of the total stimulus sentences were not understood as uttered by the interviewer. The reason why this type of error was not detected in the interview was because the participants gave responses to these stimulus sentences based on the parts of the question they understood. The second error type was misinterpretation of the polarity of the expected answer. Although not large in number, there were several 
cases where the subjects interpreted the polarity of the proposition opposite from what it was. The fact that most of the errors were observed in NQs with a positive expected answer indicates a possible influence from Japanese. That is, as presented in Chapter 2, the errors can be attributable to the L1 because the default interpretation of the Japanese NQs has a negative proposition. Although these two types of errors did not trigger miscommunication in the interview, it is worthwhile delving into the possible interaction of the actual performance and internal process. The overall results of the retrospective analysis are presented in table 5.

The following section presents the detailed discussion of the results of this study. 


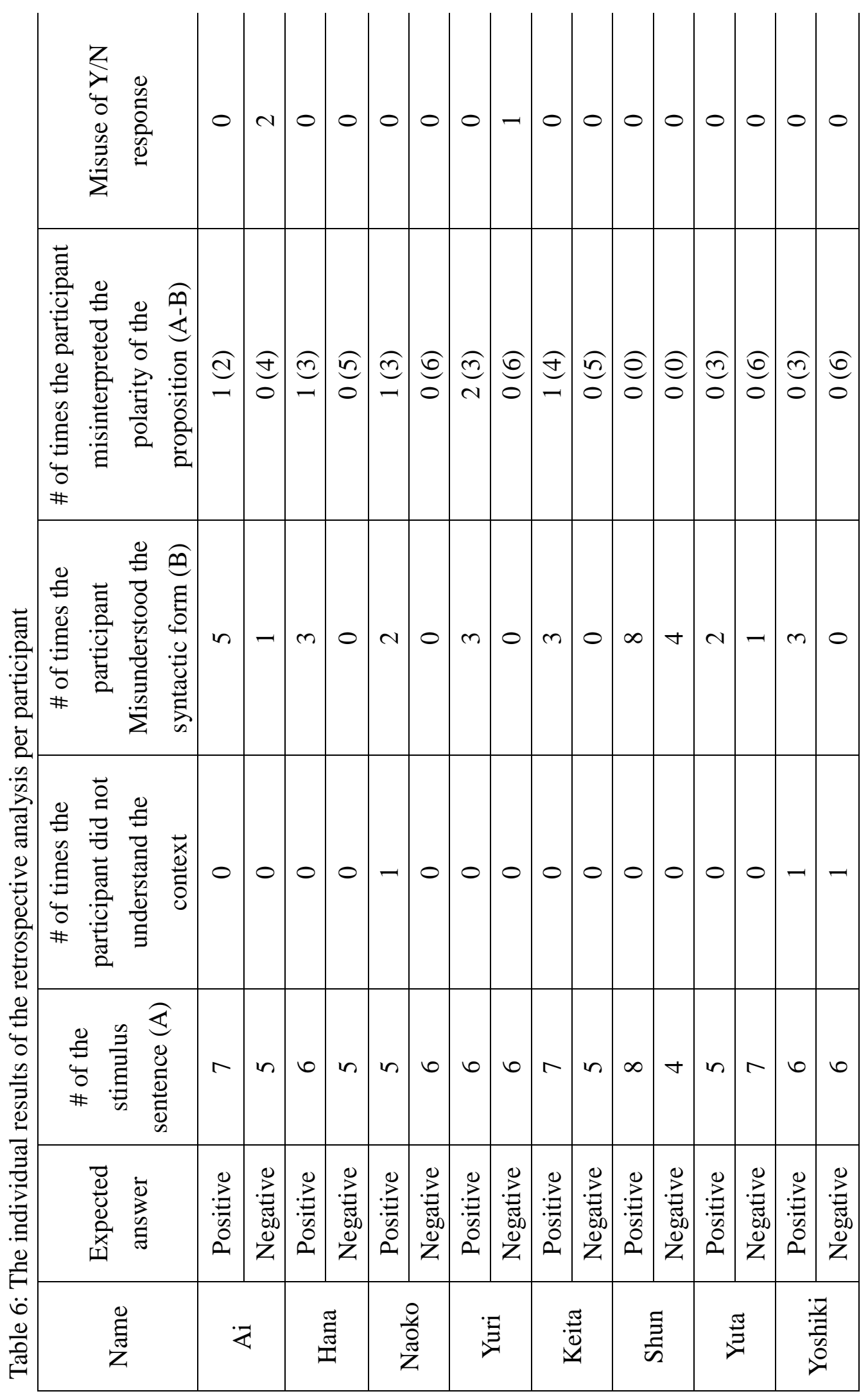




\section{Discussion of the results}

\section{Misuse of the Y/N response}

This study elicited only 3 tokens of incorrect $\mathrm{Y} / \mathrm{N}$ responses out of 94 total responses. While this rate of errors seems negligible, it is nevertheless of our interest to investigate the source of the errors.

In this study, two stimulus sentences elicited an incorrect $\mathrm{Y} / \mathrm{N}$ response. These are the following two stimulus sentence (from Ai's interview):

8) I: Which one do you like better?

S: If I can speak English well, I like American classes.

I: You can speak English good. So, don't you like classes where you just listen and don't really participate?

S: *Yeah.

9) I: What were the classes you took?

S: One is English, and one is about environmental sustainability, and one is African history.

I: Sounds cool. So, was your English class the special Waseda class?

S: Yeah, special Waseda.

I: So, didn't you take any IELP class?

S: *Yeah.

As described in the section above, the two stimulus sentences have a negative expected answer because the prior discourse explicitly induces the interviewer to have a negative assumption about the proposition in question. Two participants, Ai and Yuri, made an incorrect $\mathrm{Y} / \mathrm{N}$ response to the stimulus sentence in (8), and Ai made an incorrect $\mathrm{Y} / \mathrm{N}$ response to the stimulus sentence in (9). As discussed above, it is quite likely that the L1 influenced their responses.

A question arises, however, as to why their $\mathrm{Y} / \mathrm{N}$ responses showed variation depending on the stimulus sentences. Limiting the discussion to the NQs that have a negative expected answer, Ai and Yuri received five and six stimulus sentences, 
respectively. However, the errors were observed only for the stimulus sentences in (8) and (9). In order to look into the variation, observe the following dialogue taken from Ai's interview:

10) I: What kind of activity did you have in the class?

S: Only he talks about the topics, but sometimes students explained their opinions, and there are a little bit discussion, and I also watched some movie. I: Okay. So, didn't you have any group work in the class?

S: No. (we didn't).

In this dialogue, $\mathrm{Ai}$ understood both the prior discourse and the stimulus sentence correctly. The stimulus sentence is similar to the ones in (8) and (9) in that in the prior discourse, the participant's utterance restricted the possible polarity of the proposition in the question. However, Ai made a correct $\mathrm{Y} / \mathrm{N}$ response for this stimulus sentence in (10).

The difference between these stimulus sentences possibly lies in the strength of the assumption. Although throughout this study, assumptions were described as either positive or negative, it is obvious that assumptions are not black-and-white (Bublitz, 1981; Dusvoka, 1981). If we try to interpret the polarity of the stimulus sentences the other way around, (10) sounds more natural than the other two because there is no specific context that prevents the interviewer from having a positive expected answer. For the other two stimulus sentences, there is prior discourse where the participant's statement quite clearly indicates the polarity of the expected answer.

The assumption toward a certain proposition is always relative, and it may be possible that the stronger negative assumption that the questioner holds toward the stimulus sentence, the more likely a NQ will elicit an incorrect $\mathrm{Y} / \mathrm{N}$ response. In other 
words, there would be a correlation between the strength of the negative assumption and the possibility of eliciting a misuse of a $\mathrm{Y} / \mathrm{N}$ response.

If this account holds true, it is easy to estimate when an error of the $\mathrm{Y} / \mathrm{N}$ response can take place. Compare the following invented-dialogues:

11) A: I am soaked with rain.

B: What? Didn't you check the weather forecast?

12) A: I am soaked with rain.

B: You didn't check the weather forecast, did you?

Both of the stimulus sentences in (11) and (12) have a negative proposition that the prior discourse causes. The difference between the two stimulus sentences is the syntactic structure; whereas the question is in interrogative in (11), a tag question is used in (12). A syntactic structure is a means to express the questioner's perspective, and the stimulus sentence in (12) has a stronger assumption towards the proposition. Based on the results of this study, one would predict that the stimulus sentence in (12) is more likely to elicit the misuse of a $\mathrm{Y} / \mathrm{N}$ response.

The interview in this study elicited two other notable behaviors in some participants' responses to the interviewer's utterances. The first intriguing behavior was observed in Yoshiki's interview. In the interview, he used yeah at inappropriate times. Following are examples of this behavior:

13) I: Didn't you take any IELP classes?

S: Yeah. Ah, no.

14) I: Do you think that the English classes you took in Japan helped you with your conversation skills?

S: Yeah, I don't think so.

In both of these cases, Yoshiki explained that he used yeah as a backchannel 
expressing his understanding of the stimulus sentence. He explained that yeah is his verbal habit and he uses it every so often. This verbal behavior is obviously misleading, and if it is behavior shared by other learners, it could be one of the reasons Japanese learners of English are frequently observed to make incorrect $\mathrm{Y} / \mathrm{N}$ responses.

The second point that deserves discussion is that the interview data show some deviation from the English norm for responses to NQs. As mentioned above, Naoko realized during the interview that the study was aimed at investigating responses to English NQs. In the retrospective protocol analysis, she stated that she had had many miscommunications involving $\mathrm{Y} / \mathrm{N}$ responses, and she did her best to match the polarity of her $\mathrm{Y} / \mathrm{N}$ response to the tag that she used. As a result, there were several cases in her responses where the syntactic forms between the question and answer did not match. Following is one such case:

15) I: Didn't you have any lectures in that class?

S: Yes, there is lectures.

As this dialogue indicates, her response showed a different syntactic structure from that of the stimulus sentence. She explained that paying attention to the polarity of a Y/N response often confuses her, and she had some experiences where the use of the tag confused her interlocutor. She therefore just focused on consistency between her Y/N choice and her own sentence. Although this behavior did not cause miscommunication in this study, it may be one of the learning pitfalls with respect to responding to NQs. 


\section{The role of context}

Context is one important factor for determining the expected answer for the stimulus sentence. This study, having a speech topic familiar to the participants, enabled the participants to understand the context without much difficulty. If a learner properly understood the prior discourse, it was far easier to correctly understand the expected answer of the stimulus sentence. Regardless of their comprehension of the context, however, this study elicited 6 misinterpretations of the polarity of the expected answer. This section attempts to diagnose why the errors occurred.

Because all the stimulus sentences in this study were interrogative structures, it is reasonable to consider the error in relation to the context. Observe the two stimulus sentences that elicited a misinterpretation of the expected answer (a replication of examples (6) and (7)):

16) I: Was last term your first term here at PSU?

S: Yes, it was my first term.

I: Wasn't it hard for you to take classes in English?

S: Yeah, of course.

17) I: Do you like discussions?

S: If I can speak English well, I like it.

I: I heard that many Japanese don't like discussions, but isn't it nice to have discussions and exchange opinions with other students?

S: Yeah.

Five of the participants misinterpreted the expected answer for the stimulus sentence in (16), and only one participant for that in (17). The difference in the number can be explained by the fact that the prior discourse in (17), especially the existence of 'but,' specifies the polarity of the expected answer quite clearly. The fact that there was still 
a participant who misinterpreted the polarity for the stimulus sentence in (17) suggests that Japanese had an influence.

The stimulus sentence in (16), on the other hand, does not contain an explicit clue to specify the polarity of the proposition. It seems more natural that the interviewer has a positive assumption because the first term in a foreign school is usually hard, but five out of seven participants understood the stimulus sentence to have a negative expected answer (one participant did not understand the question correctly).

This behavior indicates a possibility that Japanese learners of English interpret a NQ as having a negative expected answer when the prior discourse does not explicitly indicate polarity of the expected answer. This hypothesis is reasonable because, as described in Chapter 2, Japanese NQs by default have a negative proposition. It is highly possible that L1 influences not only production but also interpretation.

The stimulus sentences in this study contained another NQ that was given in a similar manner. This stimulus sentence, too, was not preceded by discourse that specified the polarity of the expected answer:

18) I: How many classes did you take last term?

S: I took three classes.

I: Don't you think taking three classes is too much?

S: I don't think so.

Although the context of (18) is quite similar to that of (16), all the participants correctly understood the polarity of the proposition, i.e., positive. Should this result be taken as counterevidence to the hypothesis above? The discrepancy of interpreted 
polarity between (16) and (18) is of great interest.

From the perspective of L1 influence, two possible explanations are conceivable as to why the discrepancy was found for the two NQs given in a similar manner. First of all, the difference in the syntactic form might have caused the different interpretations. The syntactic structure of the stimulus sentence in (18) has a complement clause that is structured positively. It is possible that participants' $\mathrm{Y} / \mathrm{N}$ response is directed to this complement clause. In other words, it is possible that their response is not "Yes, I do." but "Yes, it is." As introduced in chapter 2, the negative morpheme outside a proposition in Japanese renders the question conducive towards the proposition. Therefore, the similarity of the structure between the stimulus sentence in (18) and Japanese questions that are positively conducive might have led the participants to interpret the question to have a positive proposition. Secondly, the difference might have been caused by the verb used in the stimulus sentence. Kuno (1972) argues that the verb omou 'to think' in Japanese presupposes a positive response by default when used in NQs. Therefore, the semantic feature of the verb might have helped participants to correctly comprehend the polarity of the expected answer in (18).

If either of the two explanations or a combination of them is true, it is quite possible that the Japanese learners of English would interpret the polarity of the expected answer based on the L1 norm. Their interpretation of the expected answer is to a great extent based on the context. Therefore, if the prior discourse explicitly signals the expected answer of a stimulus sentence, the Japanese learners correctly interpret the expected answer of the NQ. If the context does not help the interpretation 
of NQs, there seems to be a proclivity for the Japanese learners of English to interpret the stimulus sentences as having a negative proposition, which is consistent with Japanese.

The low performance of the participants in Takashima's (1989) study supports this hypothesis. In his study, more than $50 \%$ percent of the participants made incorrect Y/N responses to the stimulus sentence "Don't you like English?" The Y/N responses between Japanese and English are different only when a NQ has a negative expected answer, so it is quite likely that the participants interpreted the stimulus sentence to have a negative expected answer. The fact that the stimulus sentence is given without context makes it impossible to refute the argument that the L1 influences the interpretation of the NQs.

Nevertheless, this account does not hold true for every stimulus sentence because some Japanese NQs can have a positive proposition by default. If a certain feature of English NQs, such as syntactic structure or a semantic property, overlaps with the Japanese NQs that have a positive proposition by default, the stimulus sentence would probably be interpreted as having a positive proposition. This hypothesis is supported by the discrepancy of the interpretation between stimulus sentences (16) and (18).

\section{Possible variables influencing the $\mathrm{Y} / \mathrm{N}$ response}

This section presents some of the possible variables that seemed to have influenced the performance of the participants. So far, the discussion of the results solely focused on the L1 influence. In fact, the error analysis of the Y/N responses and 
the interpretation of the polarity support the interpretation that L1 seems to be the most plausible explanation. Nevertheless, the varied results of the present study call for further consideration of other variables.

\section{Proficiency}

Many applied linguistics studies have sought to examine the relationship between a certain linguistic performance and proficiency. This study was not designed to investigate the influence of TL proficiency, but the results are consistent with the influence of it.

First of all, higher TL proficiency would increase the possibility of a participant comprehending the context, which can then help the participant give a correct $\mathrm{Y} / \mathrm{N}$ response. As it turned out in this study, the interpretation of the expected answer for the participants corresponded to the L1 to a great extent. When the prior discourse explicitly indicated the polarity of the expected answer, the participants' interpretation correctly matched with the polarity. Without an explicit clue of the expected answer, however, they interpreted a stimulus sentence as having a negative proposition. When a NQ has a negative proposition, the Japanese $\mathrm{Y} / \mathrm{N}$ response conflicts with that of English. That is, misinterpreting a NQ as having a negative proposition increases the possibility of eliciting an incorrect $\mathrm{Y} / \mathrm{N}$ response.

Nevertheless, a rather contradicting generalization can also be possible. That is, the learner with lower proficiency is more likely to miss the negative morpheme in the stimulus sentence. Considering the TOEFL score as an indicator of proficiency, the performance of the subject whose proficiency is the lowest, Shun, is intriguing; he had 
no incorrect responses. However, in the retrospective protocol analysis, he explained that he understood all the stimulus sentences as a positive question. His seemingly proficient performance was actually due to his lack of attention to the negative morpheme. It seems plausible that lower level learners pay more attention to the content words. Those who missed the negative morpheme in the stimulus sentence will produce a correct $\mathrm{Y} / \mathrm{N}$ response without difficulty because the answer to positive questions is parallel between the two languages.

\section{TL exposure}

This study is not designed to address TL exposure, but it is still an important consideration in the interlanguage construction process, language learners reconstruct their internal system through exposure to the TL. In the retrospective protocol analysis, most of the participants expressed difficulty in overcoming the misuse of $\mathrm{Y} / \mathrm{N}$ responses. Different from the environment where they originally learned English, chances are everywhere to try out their TL knowledge. As a matter of fact, six out of the eight subjects in this study said that their $\mathrm{Y} / \mathrm{N}$ responses have confused an interlocutor many times. Receiving such negative feedback to their interlanguage would be one of the strongest factors that help them to make progress towards the TL norm. One reason why the participants of this study showed high performance would probably be owing to their TL experience. Regardless of the TL proficiency, having opportunities to actually use the language would increase the accuracy of their $\mathrm{Y} / \mathrm{N}$ production. 


\section{Summary: Answers to the research questions.}

This chapter has discussed the results of the two elicitation sessions. Analyzing answers by the participants suggests answers to the two research question. For the first research question "how do Japanese learners of English choose yes or no in responding to English negative interrogatives?" the following generalizations are plausible:

- The participants respond to English NQs so that their answers are fairly consistent with the English norm.

- When a negative question has a negative expected answer, it is possible that the L1 interferes with their $\mathrm{Y} / \mathrm{N}$ response.

- The stronger the expectation for a negative answer, the more likely that the NQ will elicit an incorrect $\mathrm{Y} / \mathrm{N}$ response.

The results of the retrospective protocol analysis suggest the following generalizations regarding the second research question "how do Japanese learners of English interpret the polarity of the expected answer of English NQs?”:

- The participants appear to interpret the polarity of the expected answer fairly consistently with the English norm. When a NQ is given without context, however, it is quite likely that Japanese learners of English interpret the stimulus sentence as having a negative expected answer, which is consistent with the Japanese norm. 


\section{Chapter 5: Conclusion}

This chapter presents the conclusions of this thesis study. First, a summary of the findings and interpretations are presented. Second, limitations of the present study as well as implications for future studies are discussed. At the end of the chapter, the pedagogical implications are presented.

\section{Summary of the study}

This study was designed to address two research questions. The first research question was "how do Japanese learners of English choose yes or no in responding to English negative interrogatives?”

The results of the interview indicated that the participants in this study choose a $\mathrm{Y} / \mathrm{N}$ response fairly close to the English norm. Contrary to the previous studies that found frequent errors in $\mathrm{Y} / \mathrm{N}$ responses, this study found an incorrect $\mathrm{Y} / \mathrm{N}$ response in only about $3 \%$ of the cases. The small number of errors in $\mathrm{Y} / \mathrm{N}$ responses is counter-evidence to the full-transfer theory with respect to answering the NQs. That is, when a Japanese learner of English receives a NQ, it is not always the case that their $\mathrm{Y} / \mathrm{N}$ response contradicts with the English norm.

Nevertheless, the error analysis of the results in this study indicated a possible influence from Japanese. Looking into the three tokens of an incorrect $\mathrm{Y} / \mathrm{N}$ response and the associated stimulus sentences, it was found that the errors reflect features of the Japanese NQ and a concomitant $\mathrm{Y} / \mathrm{N}$ response in two ways. First, the syntactic structures of the incorrect responses were either yes followed by a negative tag or yes by itself intended as an agreement to the negative proposition. This response is not 
acceptable in English but is acceptable in Japanese, and thus it is a reasonable indicator that the response is influenced by Japanese.

Secondly, all the stimulus sentences that elicited an incorrect response had a negative expected answer, to which the $\mathrm{Y} / \mathrm{N}$ response is different in Japanese and English. No error was observed for NQs that had a positive expected answer. These results support the hypothesis that the $\mathrm{L} 1$ is the trigger for the misuse of $\mathrm{Y} / \mathrm{N}$ responses.

In addition to L1 influence, this study further suggests another factor that may explain why Japanese learners of English are frequently found to make errors in $\mathrm{Y} / \mathrm{N}$ responses - second language proficiency. Looking into their performance and retrospective analysis, I found that general English proficiency affected the choice of Y/N responses. The results of the study suggest that there were many cases where the participants did not correctly understand the stimulus sentences. The misunderstandings occurred for several reasons, e.g., lack of listening skills, vocabulary, and grammar, to name a few. Therefore, the interference of the L1 is not likely to be the only trigger for miscommunication with NQs, but general language proficiency plays a role as well.

From the error analysis in this study, implications can be drawn as to the probability of eliciting an incorrect $\mathrm{Y} / \mathrm{N}$ response. I found an association between incorrect $\mathrm{Y} / \mathrm{N}$ responses and the strength of the interviewer's assumption. The participants received NQs in different contexts, and the errors were observed only when prior discourse explicitly dictated the interviewer's negative assumption concerning the stimulus sentences. The assumption towards a certain proposition is 
always relative, and it appeared in these data that the stronger negative assumption the questioner has, the more likely a Japanese learner of English is to make an incorrect $\mathrm{Y} / \mathrm{N}$ response.

The retrospective protocol analysis of the interview answered the second research question: How do Japanese learners of English interpret the polarity of the expected answer of English NQs?

Given the results of the translation task, it appears that the participants' interpretations of the polarity of the stimulus sentence are fairly close to the English norm. Although the default interpretation of the Japanese NQs is that the questioner has a negative assumption toward the proposition in question, the participants' interpretation of the English stimulus sentences was quite sensitive to the prior discourse. That is, they do not automatically interpret an English NQ as having a negative expected answer. With the help of context, for example, Japanese learners of English can interpret a stimulus sentence "Don't you like baseball?" as having either a positive expected answer or a negative expected answer. In this study, the participants generally showed a sophisticated performance on the interpretation of the stimulus sentences probably because the context, especially the speech topic, was familiar to them.

Nevertheless, the results of the translation task also showed a possible influence from Japanese. The task elicited six misinterpretations of the stimulus sentences. The error analysis revealed that participants' interpretation matched with the Japanese default interpretation. Namely, they misinterpreted the NQs having a positive expected answer as ones having a negative expected answer. One hypothesis 
drawn from the results is that Japanese learners of English tend to interpret a NQ as having a negative expected answer when a positive expectation is not obvious from the context.

This account does not hold true, however, when the stimulus sentence contains a certain feature that can affect the polarity of the proposition in Japanese, such as a syntactic structure or semantic feature. This study used the stimulus sentence "Don't you think that ... ?" given without specific prior discourse indicating the expected answer. The hypothesis above predicts that the learners would misinterpret the stimulus sentence as having a negative proposition, but the actual performance showed that all the participants interpreted the polarity correctly. The performance is unsurprising because the syntactic and/or semantic properties of the stimulus sentence produce a positive expected answer in Japanese by default.

From the limited number of participants and stimulus sentences given, it is not reasonable to draw conclusions about the degree of L1 influence. However, the present study has shown that there are two learning pitfalls in the acquisition of English NQs, and the fact that all the observed errors reflect the Japanese interpretation and use of $\mathrm{Y} / \mathrm{N}$ is a solid indicator that Japanese is in some way involved in the English learning process.

In conclusion, this study has shown that Japanese learners of English do not make incorrect $\mathrm{Y} / \mathrm{N}$ responses as often as reported in previous literature. With the help of the context, NQs with a positive expected answer are quite likely to elicit a correct Y/N response. Even for NQs with a negative expected answer, errors were not observed as often as transfer-based learning theory would predict. Some possible 
explanations have been discussed, such as TL exposure and proficiency, but the strength of the questioner's assumption was offered as the key explanation for the misuse of the $\mathrm{Y} / \mathrm{N}$ response. This account further predicts that different syntactic forms, like a negative declarative with a tag, would be more likely to elicit an incorrect Y/N response because the stimulus sentence itself expresses the negative proposition quite directly. The results of this study imply that the L1 influence does not explain the whole picture of the Japanese learners' interlanguage construction but is one indicator of when the errors are likely.

\section{Limitations of the study and suggestions for further research}

The present study attempted to analyze Japanese learners' spontaneous response patterns and analyze their processes in producing the responses. The strength of the research methodology is that it administered two elicitation sessions. The first session was the oral interview. Different from questionnaires or video tasks, having oral interviews enabled research of the learners' unmonitored response patterns and enhanced the credibility of the present study. The second session, the retrospective analysis of the interview, was necessary to look into the internal process of the learners' performance.

However, there are two main limitations of this research study. First, the number of participants was very low. Different from research studies that administer questionnaires or video tasks, which can be administered to large population, this study had only eight participants. The time-consuming nature of individual interviews 
limits the number of participants, and studies with more participants are needed to reach more generalized results.

The other limitation concerns the validity of the translation task used in the study. As pointed out in chapter 2, the polarity of the expected answer is a key factor that triggers miscommunication in interactions with NQs. In order to assess the interpreted polarity of the stimulus sentence, a translation task for each stimulus sentence was administered in the present study. One advantage of this task is that we can understand participants' interpretation of polarity in a clear-cut manner. However, there are two possible problems with the translation task. One is that it requires careful recall by the participants. Even with the help of a video of the interview, it is possible that their interpretations of the stimulus sentences were contaminated by their raised awareness of the stimulus sentences and the time lag. The other potential problem is that any translation task forces the participants to rely on the Japanese translation for interpretation of the stimulus sentences where, in real interactions in English, the learners may process and produce the language without any translation. Thus, it is possible that the translation task forced them to choose interpretations that did not accurately reflect their real interpretations. In similar studies in the future, it might be better if a simple scale questionnaire assessing the degree of the questioner's assumption is given to participants.

\section{Pedagogical Implication}

Negative questions are one of the most notoriously difficult grammar points 
for Japanese learners of English. A great deal of care should be taken to explain this grammar point.

Having described how the two languages are different and investigated the participants' performances, I suggest that we should be explicit in teaching how the two languages are different. This study has shown the detailed differences between the two languages, which include not just $\mathrm{Y} / \mathrm{N}$ responses but also the stimulus sentences. Introducing the differences would raise learners' awareness and be of great help when they respond to NQs. It is undoubtedly true that learners modify their interlanguage through experience. Knowing how the two languages are different can help learners to understand negative feedback they receive and to restructure their interlanguage accordingly.

In order to avoid miscommunication, there are some tips that English teachers can implement in their classes. First of all, teachers should repeatedly teach students that responding only yes or no is problematic. In fact, the miscommunication that occurred in the present study could have been avoided if the participants added a tag to their yes or no response. Although it is often the case with an elementary level classes that teachers correct students $\mathrm{Y} / \mathrm{N}$ responses if uttered without a tag, this teaching method is not usually carried over in a higher level of education when students become conscious of "authentic" English. In order to avoid miscommunication, it is vital to pay close attention to an entire utterance. It is another helpful strategy that teachers ask for clarification when students $\mathrm{Y} / \mathrm{N}$ responses are not clear so that students realize there is a problem.

Another teaching strategy is to present two translation patterns for an English 
NQ. As exemplified in the translation task, English NQs can be translated into two ways in Japanese. If teachers present the two translations and ask how students would answer the stimulus sentences, teachers could explain that the Japanese Y/N response is variable depending on stimulus sentences. As described above, awareness-raising could be a very effective approach in teaching English NQs. Several strategies to raise awareness towards this grammar point are conceivable, but because the L1 seems to be the most plausible explanation for the frequently observed errors, I suggest that we explicitly mention how learners get confused from the perspective of Japanese.

In sum, this study has shown a possible picture of how L1 influences the acquisition of English NQs. Different from the simple equation that observing an error equals interference from the L1, this study provided a sound basis to pinpoint how the L1 influences the TL use. Especially, the study's contribution to shed light on stimulus sentences is of great benefit for future research. Because the choice of Japanese Y/N responses is dependent on the polarity of the stimulus sentence, it is necessary to look into both the $\mathrm{Y} / \mathrm{N}$ response and the stimulus sentence. As a matter of fact, the results of the present study showed that stimulus sentences are one solid indicator of when an error is likely. Now that it has been pointed out how the L1 could influence the acquisition of NQs, I hope that future studies focus on investigating the degree of L1 influence with more participants and in a variety of registers.

The present study made clear the picture of how L1 interferes with the acquisition of NQs. With the solid comparison of the two languages, this study enabled pinpointing the source of errors observed in responding to NQs. Especially, the finding that errors are likely when a questioner has a strong negative assumption 
towards the proposition is quite meaningful for EFL teachers. It gives ideas for how to raise their awareness in teaching as well as how to make a test assessing this grammar point. Negative questions are a notorious grammar point that will always give learners of English difficulty. We, as language educators, can help them better by truly understanding their problems. 


\section{References}

Anderson, R. (1984). The one to one principle of interlanguage construction. Language Learning, 34, 77-95.

Angel, J., Nakamura, A., \& Nakamura, M. (2000). Japanese responses hai, ee, and un: yes, no, and beyond. Language \& Communication, 20, 55-86.

Akiyama, M. (1976). Negative questions in Japanese-speaking, English-speaking, and Japanese-English bilingual children. Papers and reports on child language development, 12, 23-30.

Akiyama, M. M. (1979). Yes-no answering systems in young children. Cognitive Psychology, 11, 485-504.

Baik, M., \& Shim, R. (1993). Yes, we have no bananas: English native tags in cross-linguistic communication. Studies in the Linguistic Sciences, 23, 43-59.

Bublitz, W. (1981). Conducive yes-no questions in English. Linguistics, 19, 851-870.

Dulay, H., \& Burt, M. (1974). Natural sequences in child second language acquisition. Language Learning, 24, 37-53.

Duskova, L. (1981). Negative questions in English. International Review of Applied Linguistics in Language Teaching. 19, 181-194.

Ellis, R. (1985). Understanding second language acquisition. Oxford: Oxford University Press.

Ellis, R. (1994). The study of second language acquisition. Oxford: Oxford University Press.

Gass, S. (1997). Input, interaction, and the second language learner. New Jersey: Lawrence Erlbaum Associates.

Green, P., \& Hecht, K. (1992). Implicit and explicit grammar: An empirical study. Applied Linguistics, 13, 168-184.

Jarvis, S. (2000). Methodological rigor in the study of transfer: Identifying L1 influence in the interlanguage lexicon. Language Learning, 50, 245-309.

Kang, S., \& Lim-Chang, Y. (1998). No, I am a student: A study of linguistics and pragmatic transfer in SLA. SECOL Review, 22, 106-159

Kuno, S. (1973). The structure of the Japanese language. Cambridge, Mass: MIT Press. 
Lado, R. (1957). Linguistics across cultures: Applied linguistics for language teachers. Ann Arbor: University of Michigan Press.

Lane, C, L. (1993). Yes, I don't understand: yes, no and European-Polynesian miscommunication in New Zealand. Journal of Pragmatics, 20, 163-188.

Martin, S, E. (1962). Essential Japanese. Tokyo: Carles E. Tuttle Co.

Morita, T. (1988). Rinkyoshin to nihonjin, nihonbunkaron (The ad hoc council on Education and the studies on the uniqueness of the Japanese). Tokyo: Shin Nihon Shuppansha.

Nagao, J. (2005). Some difficulties in responding to negative polar interrogatives and negative declaratives in English and pedagogical implications for Japanese EFL learners. Dissertation Abstract international. 66(6), 2194-A-2195-A. (UMI No.DA3179580)

Odlin, T. (1989). Language transfer: Cross-linguistic influence in language learning. Cambridge: Cambridge Press.

Pope, E. (1976). Questions and answers in English. Mouton.

Shibatani, M. (1972). Negative questions and conveyed meaning. Papers in Japanese Linguistics, 1, 321-323.

Takashima, H. (1989). How Japanese learners of English answer negative yes-no questions: A case of language transfer. International Review of Applied Linguistics in Language Teaching, 27, 113-124.

Teramura, H. (1984). The syntax and meaning of Japanese. Tokyo: Kurosio Publishers.

Taylor, B. (1975). The use of overgeneralization and transfer learning strategies by elementary and intermediate students of ESL. Language Learning, 25, 73-107.

Yat-shing, C. (1974). Negative questions in Chinese. Journal of Chinese Linguistics, 2, 325-339. 


\section{Appendix}

Interview Scenario (S: student, I: interviewer)

Major

$1^{\text {st }}:$ How many classes did you take last term?

S: (I took) \# classes/ I took A, B, and C.

I: (so, you took \# classes.) (How many days a week did you have classes?)

Don't you think taking \# classes is too much?

$\mathrm{S}: \mathrm{Y} / \mathrm{N}$ or just states opinion.

Don't you think taking 5 days of class a week is too much?

I: comment

$2^{\text {nd }}$ : What were the classes?/ Did you say you took A, B, and C?

S: (I took) A, B, and C.

$\square \begin{aligned} & \frac{(O h,)}{\text { Didn't you take D? }} \\ & (D=\text { regular class, grammar/writing, listening, pronunciation, }\end{aligned}$

$\underline{\text { etc) }}$

$\mathrm{S}: \mathrm{Y} / \mathrm{N}$, or else

I: comment

$3^{\text {rd }}$. Which class did you like the best (or better)?

S: I like A.

$\mathrm{I}$ : (Oh, I heard that A in PSU is a pretty tough class.)

(That sounds difficult)

\section{Wasn't that difficult for you?}

$\mathrm{S}: \mathrm{Y} / \mathrm{N}$

I: comment

$4^{\text {th }}$ : Could you explain why you liked the class? (distracter)

S: Because...

I: comment

$5^{\text {th }}$ : What kind of activities did you do in the class (another class if more natural)?

S: A, B, C, etc...

(So, ) didn't you have D in the class?

( $D=$ pop quiz, discussion, pair work, group work, etc...)

$\mathrm{S}: \mathrm{Y} / \mathrm{N}$, or else.

I: comment

$6^{\text {th }}$. What was the main activity in the class?

S: A, B, etc...

I: (But, for your learning in general,)

Wouldn't it had been better if you had D more? 
$\underline{(D=\text { pop quiz, discussion, pair work, group work, etc...) }}$

$7^{\text {th }}$ : Do you like discussion?/ (possibly) you said you don't like discussion, right?

$\mathrm{S}: \mathrm{Y} / \mathrm{N}$

I: (But) I heard that many Japanese students don't like discussion.

(But) Isn't it nice to have discussion and exchange opinions?

$8^{\text {th }}$ : (By the way) Was last tem your first term at PSU?

S: Yes

S: $\mathrm{Y} / \mathrm{N}$

Wasn't it hard for you to take classes in English?

I: Comment

$9^{\text {th }}$ : What was the most difficult thing classes in English? (distracter)

S: listening or speaking (possibly, reading or writing)

Japanese ELT

$10^{\text {th }}$ : When you studied English in Japan, what did you study?

S: A, B, C, etc...

(So) Didn't you study D?

(D= listening, speaking, writing, sound symbols, etc...)

S: Y/N, or else.

I: comment

$11^{\text {th. }}$ Were the classes taught in English?

$\mathrm{S}: \mathrm{Y} / \mathrm{N}$

S: $\mathrm{Y} / \mathrm{N}$

Wouldn't it have been better if the teachers use more English?

I: comment

$12^{\text {th }}$ : Do you think that the English classes you took in Japan helped you with your conversation skills?

S: No

I: Kosuke also thinks that Japanese English education is not that effective in improving practical communication (possibly academic writing) skill.

Don't you hope Japanese English education focuses more on communication in the future?

Or

S: Yes

I: Oh, really? Kosuke thinks Japanese English education is not that effective in improving communication skills.

Don't you agree with him?

Classes in general

$13^{\text {th }}$ : How are classes in Japan different from here? 
S: Importance of participation, class activities, h.w., etc...)

Japanese classes

American classes

I: Which one do you like better?

S: Japanese classes/ American classes

Don't you like D?

(D=participating in class, doing the homework, or else)?

At the end

$14^{\text {th }}$ : Your English is very good!!

(Yes)Wasn't this interview easy for you?

Or

$14^{\text {th }}$ : Did you bring this questionnaire with you?

S: No

(No)Didn't Kosuke tell you to bring it? 